\title{
Composite Quasar Spectra from the Sloan Digital Sky Survey
}

\section{Citation}

Vanden Berk, Daniel E., Gordon T. Richards, Amanda Bauer, Michael A. Strauss, Donald P. Schneider, Timothy M. Heckman, Donald G. York, et al. 2001. "Composite Quasar Spectra from the Sloan Digital Sky Survey." The Astronomical Journal 122 (2) (August): 549-564. doi:10.1086/321167.

\section{Published Version}

doi:10.1086/321167

\section{Permanent link}

http://nrs.harvard.edu/urn-3:HUL.InstRepos:33461905

\section{Terms of Use}

This article was downloaded from Harvard University's DASH repository, and is made available under the terms and conditions applicable to Other Posted Material, as set forth at http:// nrs.harvard.edu/urn-3:HUL.InstRepos:dash.current.terms-of-use\#LAA

\section{Share Your Story}

The Harvard community has made this article openly available.

Please share how this access benefits you. Submit a story.

\section{Accessibility}




\title{
COMPOSITE QUASAR SPECTRA FROM THE SLOAN DIGITAL SKY SURVEY ${ }^{1}$
}

Daniel E. Vanden Berk, ${ }^{2}$ Gordon T. Richards, ${ }^{3}$ AMANda Bauer, ${ }^{4}$ Michael A. Strauss, ${ }^{5}$ Donald P. Schneider, ${ }^{3}$ Timothy M. Heckman, ${ }^{6}$ Donald G. York, ${ }^{7,8}$ Patrick B. Hall,${ }^{5,9}$ XiaOhul Fan, ${ }^{5,10}$ G. R. KNapP, ${ }^{5}$

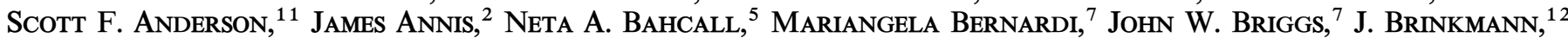
Robert Brunner, ${ }^{13}$ Scott Burles, ${ }^{2}$ Larry Carey, ${ }^{11}$ Francisco J. Castander,,${ }^{7}{ }^{74}$ A. J. Connolly, ${ }^{15}$ J. H. Crocker, ${ }^{6}$ István Csabai,${ }^{6,16}$ Mamoru Doi,${ }^{17}$ Douglas Finkbeiner, ${ }^{18}$ Scott Friedman, ${ }^{6}$ Joshua A. Frieman, ${ }^{2,7}$

Masataka Fukugita, ${ }^{19}$ James E. GunN, ${ }^{5}$ G. S. Hennessy, ${ }^{20}$ Željko Ivezić, ${ }^{5}$ Stephen Kent, ${ }^{2,7}$ Peter Z. Kunszt, ${ }^{6}$

D. Q. LAMb,${ }^{7}$ R. French Leger, ${ }^{11}$ Daniel C. Long, ${ }^{12}$ Jon Loveday, ${ }^{21}$ Robert H. Lupton, ${ }^{5}$ Avery Meiksin, ${ }^{22}$

Aronne Merelli, ${ }^{12,23}$ Jeffrey A. Munn, ${ }^{24}$ Heidi Jo Newberg, ${ }^{25}$ Matt Newcomb, ${ }^{23}$ R. C. Nichol, ${ }^{23}$ Russell Owen, ${ }^{11}$ Jeffrey R. Pier, ${ }^{24}$ Adrian Pope, ${ }^{6,23}$ Constance M. Rockosi, ${ }^{7}$ David J. Schlegel, ${ }^{5}$ Walter A. Siegmund, ${ }^{11}$

Stephen Smee, ${ }^{6,26}$ Yehuda Snir, ${ }^{23}$ Chris Stoughton, ${ }^{2}$ Christopher Stubbs, ${ }^{11}$ Mark Subbarao, ${ }^{7}$ Alexander S. Szalay, ${ }^{6}$ Gyula P. Szokoly,${ }^{6}$ Christy Tremonti, ${ }^{6}$ Alan Uomoto, ${ }^{6}$ Patrick Waddell,${ }^{11}$ BRIAN YANNY, ${ }^{2}$ AND WeI ZHENG ${ }^{6}$

Received 2001 March 8; accepted 2001 May 2

\begin{abstract}
We have created a variety of composite quasar spectra using a homogeneous data set of over 2200 spectra from the Sloan Digital Sky Survey (SDSS). The quasar sample spans a redshift range of $0.044 \leq z \leq 4.789$ and an absolute $r^{\prime}$ magnitude range of -18.0 to -26.5 . The input spectra cover an observed wavelength range of $3800-9200 \AA$ at a resolution of 1800 . The median composite covers a restwavelength range from 800 to $8555 \AA$ and reaches a peak signal-to-noise ratio of over 300 per $1 \AA$ resolution element in the rest frame. We have identified over 80 emission-line features in the spectrum. Emission-line shifts relative to nominal laboratory wavelengths are seen for many of the ionic species. Peak shifts of the broad permitted and semiforbidden lines are strongly correlated with ionization energy, as previously suggested, but we find that the narrow forbidden lines are also shifted by amounts that are strongly correlated with ionization energy. The magnitude of the forbidden line shifts is $\lesssim 100$ $\mathrm{km} \mathrm{s}^{-1}$, compared with shifts of up to $550 \mathrm{~km} \mathrm{~s}^{-1}$ for some of the permitted and semiforbidden lines. At wavelengths longer than the Ly $\alpha$ emission, the continuum of the geometric mean composite is well fitted by two power laws, with a break at $\approx 5000 \AA$. The frequency power-law index, $\alpha_{v}$, is -0.44 from $\approx 1300$ to $5000 \AA$ and -2.45 redward of $\approx 5000 \AA$. The abrupt change in slope can be accounted for partly by host-galaxy contamination at low redshift. Stellar absorption lines, including higher order Balmer lines, seen in the composites suggest that young or intermediate-age stars make a significant contribution to the light of the host galaxies. Most of the spectrum is populated by blended emission lines, especially in the range $1500-3500 \AA$, which can make the estimation of quasar continua highly uncertain unless large ranges in wavelength are observed. An electronic table of the median quasar template is available.
\end{abstract}

Key words: quasars: emission lines - quasars: general

On-line material: machine-readable tables

${ }^{1}$ Based on observations obtained with the Sloan Digital Sky Survey, which is owned and operated by the Astrophysical Research Consortium.

${ }^{2}$ Fermi National Accelerator Laboratory, P.O. Box 500, Batavia, IL 60510.

${ }^{3}$ Department of Astronomy and Astrophysics, 525 Davey Laboratory, Pennsylvania State University, University Park, PA 16802.

${ }^{4}$ Department of Physics, University of Cincinnati, Cincinnati, OH 45221.

${ }^{5}$ Princeton University Observatory, Peyton Hall, Princeton, NJ 08544-1001.

${ }^{6}$ Department of Physics and Astronomy, Johns Hopkins University, 3701 San Martin Drive, Baltimore, MD 21218.

${ }^{7}$ Department of Astronomy and Astrophysics, University of Chicago, 5640 South Ellis Avenue, Chicago, IL 60637.

${ }^{8}$ Enrico Fermi Institute, University of Chicago, 5640 South Ellis Avenue, Chicago, IL 60637.

9 Pontificia Universidad Católica de Chile, Departamento de Astronomía y Astrofísica, Facultad de Física, Casilla 306, Santiago 22, Chile.

${ }^{10}$ Institute for Advanced Study, Olden Lane, Princeton, NJ 08540-0631; Princeton University Observatory, Peyton Hall, Princeton, NJ 08544-1001.

${ }_{11}^{11}$ Department of Astronomy, University of Washington, Box 351580, Seattle, WA 98195.

12 Apache Point Observatory, P.O. Box 59, Sunspot, NM 88349-0059.

${ }^{13}$ Department of Astronomy, 105-24, California Institute of Technology, 1201 East California Boulevard, Pasadena, CA 91125.

14 Observatoire Midi-Pyréneés, 14 Avenue Edouard Belin, F-31400 Toulouse, France.

15 Department of Physics and Astronomy, University of Pittsburgh, 3941 O’Hara Street, Pittsburgh, PA 15260.

16 Department of Physics of Complex Systems, Eötvös University, Pázmány Péter sétány 1/A, H-1117 Budapest, Hungary.

17 Department of Astronomy and Research Center for the Early Universe, School of Science, University of Tokyo, Hongo, Bunkyo, Tokyo, 113-0033, Japan.

18 Departments of Physics and Astronomy, University of California, Berkeley, 601 Campbell Hall, Berkeley, CA 94720.

${ }^{19}$ Institute for Cosmic Ray Research, University of Tokyo, Kashiwa, 2778582, Japan.

${ }^{20}$ US Naval Observatory, 3450 Massachusetts Avenue, NW, Washington, DC 20392-5420.

21 Astronomy Centre, University of Sussex, Falmer, Brighton BN1 9QJ, UK.

${ }^{22}$ Royal Observatory, Edinburgh, EH9 3HJ, UK.

${ }^{23}$ Department of Physics, Carnegie Mellon University, 5000 Forbes Avenue, Pittsburgh, PA 15232.

${ }^{24}$ US Naval Observatory, Flagstaff Station, P.O. Box 1149, Flagstaff, AZ 86002-1149.

25 Department of Physics, Rensselaer Polytechnic Institute, SC1C25, Troy, NY 12180.

${ }^{26}$ Department of Astronomy, University of Maryland, College Park, MD 20742-2421. 


\section{INTRODUCTION}

Most quasar spectra from ultraviolet to optical wavelengths can be characterized by a featureless continuum and a series of mostly broad emission line features; compared with galaxies or stars, these spectra are remarkably similar from one quasar to another. The first three principal components' spectra account for about $75 \%$ of the intrinsic quasar variance (Francis et al. 1992). Subtle global spectral properties can be studied by combining large numbers of quasar spectra into composites. The most detailed composites (Francis et al. 1991; Zheng et al. 1997; Brotherton et al. 2001) use hundreds of moderate-resolution spectra and typically cover a few thousand angstroms in the quasar rest frame. These high signal-to-noise ratio $(\mathrm{S} / \mathrm{N})$ spectra reveal variations from a single power law in the general continuum shape and weak emission features that are rarely detectable in individual quasar spectra.

The Sloan Digital Sky Survey (SDSS; York et al. 2000) already contains spectra for over 2500 quasars as of 2000 June, and by the survey's end, it will include on the order of $10^{5}$ quasar spectra. The identification and basic measurement of this sample will be done using an automated pipeline, part of which uses templates for object classification and redshift determination. As one of the first uses of the initial set of spectra, we have created a composite quasar spectrum for use as a template. The large number of spectra, their wavelength coverage, relatively high resolution, and high $\mathrm{S} / \mathrm{N}$ make the current SDSS sample ideal for the creation of composite quasar spectra. The resulting composite spectrum covers a vacuum rest-wavelength range of 800 $8555 \AA$. The peak $\mathrm{S} / \mathrm{N}$ per $1 \AA$ resolution element is over 300 near $2800 \AA$ - several times higher than the previous best ultraviolet/optical composites (see, e.g., Francis et al. 1991; Zheng et al. 1997; Brotherton et al. 2001).

In addition to serving as a cross-correlation template, the composite is useful for the precise measurement of emissionline shifts relative to nominal laboratory wavelengths, the calculation of quasar colors for improved candidate selection and photometric redshift estimates, the calculation of $K$-corrections used in evaluating the quasar luminosity function, and the estimation of the backlighting flux density continuum for measurements of quasar absorption-line systems. Composites can also be made from subsamples of the input spectra chosen according to quasar properties, such as luminosity, redshift, and radio loudness. The dependence of global spectral characteristics on various quasar properties will be the subject of a future paper (Vanden Berk et al. 2001). Here we concentrate on the continuum and emission-line properties of the global composite. We describe the SDSS quasar sample in $\S 2$ and the method used to generate the composite spectra in $\S 3$. The continuum and emission-line features are measured and discussed in $\S \S 4$ and 5. Wavelengths throughout the paper are vacuum values, except when using the common notation for line identification (truncated air values for wavelengths greater than $3000 \AA$ and truncated vacuum values for wavelengths less than $3000 \AA$ ). We use the following values for cosmological parameters throughout the paper: $H_{0}=100$ $\mathrm{km} \mathrm{s}^{-1}, \Omega_{m}=1.0, \Omega_{\Lambda}=0$, and $q_{0}=0.5$.

\section{SDSS QUASAR SAMPLE}

The spectra were obtained as part of the commissioning phase of the SDSS. Details of the quasar candidate target selection and spectroscopic data reduction will be given in future papers (Richards et al. 2001a; Newberg et al. 2001; Frieman et al. 2001). The process is summarized here. Quasar candidates are selected in the color space of the SDSS $u^{\prime} g^{\prime} r^{\prime} i^{\prime} z^{\prime}$ filter system (Fukugita et al. 1996) from objects found in imaging scans with the SDSS $2.5 \mathrm{~m}$ telescope and survey camera (Gunn et al. 1998). The effective central wavelengths of the filters for a power-law spectrum with a frequency index of $\alpha=-0.5$ are approximately 3560 , $4680,6175,7495$, and $8875 \AA$ for $u^{\prime}, g^{\prime}, r^{\prime}, i^{\prime}$, and $z^{\prime}$, respectively. Quasar candidates are well separated from the stellar locus in color space, and the filter system allows the discovery of quasars over the full range of redshifts from $z=0$ to $\approx 7$. The locations of known quasars in the SDSS color space as a function of redshift are shown by Fan et al. (1999, 2000, 2001), Newberg et al. (1999), Schneider et al. (2001), and especially Richards et al. (2001b), who plot the locations of over 2600 quasars for which there is SDSS photometry. Quasar candidates are selected to $i^{\prime} \approx 19$ in the low-redshift $(z \lesssim 2.5)$ regions of color space, and no discrimination is made against extended objects in those regions. High-redshift quasar candidates are selected to $i^{\prime} \approx 20$. Objects are also selected as quasar candidates if they are point sources with $i^{\prime} \leq 19$ and match objects in the VLA FIRST radio source catalog (Becker, White, \& Helfand 1995). Thus, quasars in the SDSS are selected by both optical and radio criteria. These data were taken while the hardware and, in particular, the target selection software was being commissioned. Therefore, the selection criteria for quasars has evolved somewhat over the course of these observations and will not exactly match the final algorithm discussed in Richards et al. (2001a). Because of the changing quasar selection criteria and the loose definition of "quasar," discretion should be exercised when using the global composite spectra generated from this quasar sample as templates for quasars in other surveys or in subsets of the SDSS quasar sample.

The candidates were observed using the $2.5 \mathrm{~m}$ SDSS telescope (Siegmund et al. 2001) at Apache Point Observatory and a pair of double fiber-fed spectrographs (Uomoto et al. 2001). Targeted objects are grouped into $3^{\circ}$ diameter "plates," each of which holds 640 optical fibers. The fibers subtend 3 " on the sky, and their positions on the plates correspond to the coordinates of candidate objects, sky positions, and calibration stars. Approximately 100 fibers per plate are allocated to quasar candidates. At least three 15 minute exposures are taken per plate. So far, spectra have been taken mainly along a 2.5 wide strip centered on the celestial equator, with a smaller fraction at other declinations. The spectra in this study were grouped on 66 plates that overlap somewhat to cover approximately $320 \mathrm{deg}^{2}$ of sky covered by the imaging survey. The plates were observed from 1999 October to 2000 June. The raw spectra were reduced with the SDSS spectroscopic pipeline (Frieman et al. 2001), which produces wavelength- and fluxcalibrated spectra that cover an observed wavelength range from 3800 to $9200 \AA$ at a spectral resolution of approximately 1850 blueward of $6000 \AA$ and 2200 redward of $6000 \AA$. These spectra and more will be made publicly available (in electronic form) in 2001 June as part of the SDSS early data release (Stoughton et al. 2001).

The flux calibration is only approximate at this time and a point that deserves elaboration, since it is the most important source of uncertainty in the continuum shapes of the 
spectra. Light losses from differential refraction during the observations are minimized by tracking guide stars through a $g^{\prime}$ filter-the bluest filter within the spectral range. Several F subdwarf stars are selected for observation (simultaneously with the targeted objects) on each plate. One of these - usually the bluest one-is selected, typed, and used to define the response function. This process also largely corrects for Galactic extinction, since the distances to the F subdwarfs employed are typically greater than 2.5 $\mathrm{kpc}$, and all of the survey area is at high Galactic latitude. Uncertainties can arise in the spectral typing of the star and from any variation in response across a plate. A check on the accuracy of the flux calibration is made for each plate by convolving the calibrated spectra with the filter transmission functions of the $g^{\prime}, r^{\prime}$, and $i^{\prime}$ bands and comparing the result with magnitudes derived from the imaging data using an aperture the same diameter as the spectroscopic fibers. For a sample of about 2300 SDSS quasar spectra, the median color difference between the photometric and spectral measurements, after correcting the photometric values for Galactic extinction (Schlegel, Finkbeiner, \& Davis 1998), was found to be $\Delta\left(g^{\prime}-r^{\prime}\right) \approx 0.01$ and $\Delta\left(r^{\prime}-i^{\prime}\right) \approx$ 0.04 . This means that the spectra tend to be slightly bluer than expectations from the photometry. For a pure powerlaw spectrum with true frequency index of $\alpha_{v}=-0.5$, which is often used to approximate quasars, the difference in both colors would result in a measured index that is systematically greater (bluer) by about 0.1 . Quasar spectra are not pure power laws, and the color differences are well within the intrinsic scatter of quasars at all redshifts (Richards et al. 2001b). In addition, the SDSS photometric calibration is not yet finalized, and the shapes of the filter transmission curves are still somewhat uncertain, both of which could contribute to the spectroscopic versus photometric color differences. The colors of the combined spectra agree well with the color-redshift relationships found by Richards et al. (2001b; see $\S \S 3$ and 5), which also leads us to believe that the flux calibrations are reasonably good. However, we caution that the results here on the combined continuum shape cannot be considered final until the SDSS spectroscopic calibration is verified.

Quasars were identified from their spectra and approximate redshift measurements were made by manual inspection. ${ }^{27}$ We define quasar to mean any extragalactic object with at least one broad emission line and that is dominated by a nonstellar continuum. This includes Seyfert galaxies, as well as quasars, and we do not make a distinction between them. Spectra were selected if the rest-frame FWHM of the strong permitted lines, such as C IV, Mg II, and the Balmer lines, were greater than about $500 \mathrm{~km} \mathrm{~s}^{-1}$. In most cases, those line widths well exceeded $1000 \mathrm{~km} \mathrm{~s}^{-1}$. Since we require only one broad emission line, some objects that may otherwise be classified as "type 2 " AGNs (those with predominantly narrow emission lines) are also included in the quasar sample. Spectra with continua dominated by stellar features, such as unambiguous $\mathrm{Ca} \mathrm{H}$ and $\mathrm{K}$ lines, or the $4308 \AA$ G-band, were rejected. This definition is free from traditional luminosity, or morphology-based criteria and is also intended to avoid introducing a significant spectral component from the host galaxies (see $\S 5$ ). Spectra with broad absorption line features (BAL quasars), which com-

\footnotetext{
${ }^{27}$ Refined redshift measurements were made later as described in $\S 3$.
}

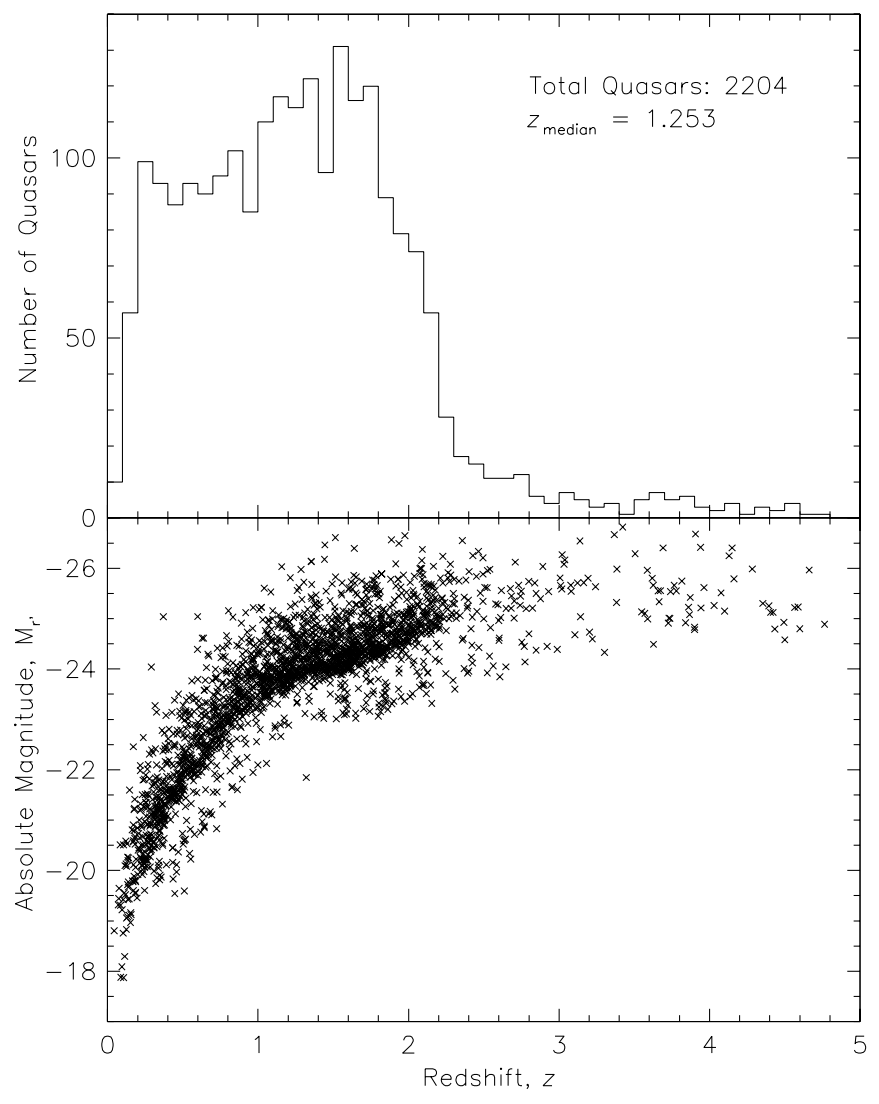

FIG. 1.-Redshift distribution of the 2204 quasars used for the composite spectra (top), and the absolute $r^{\prime}$ magnitude, $M_{r}$, vs. redshift (bottom). The median redshift is $z=1.253$.

prise about $4 \%$ of the initial sample, were removed from the input list. We are studying BAL quasars in the SDSS sample intensively, and initial results are forthcoming (see, e.g., Menou et al. 2001); the focus of the present paper is on the intrinsic continua of quasars, and BAL features can heavily obscure the continua. Other spectra with spurious artifacts introduced either during the observations or by the data reduction process (about $10 \%$ of the initial sample) were removed from the input list. ${ }^{28}$ Spectra obtained as part of SDSS follow-up observations on other telescopes, such as the high-redshift samples of Fan et al. (1999, 2000, 2001), Schneider et al. (2000, 2001), and Zheng et al. (2000), were not included. Figure 1 shows the redshift distribution of the quasars used in the composite and the absolute $r^{\prime}$ magnitudes versus redshift. Discontinuities in the selection function for the quasars, such as the fainter magnitude limit for high-redshift candidates, are evident in Figure 1. The final list of spectra contains 2204 quasars spanning a redshift range of $0.044 \leq z \leq 4.789$, with a median quasar redshift of $\bar{z}=1.253$. The vast majority of the magnitudes lie in the range $17.5<r^{\prime}<20.5$.

\section{GENERATING THE COMPOSITES}

The steps required to generate a composite quasar spectrum involve selecting the input spectra, determining accurate redshifts, rebinning the spectra to the rest frame, scaling

\footnotetext{
${ }^{28}$ These artifacts are due to the inevitable problems of commissioning both the software and hardware, and the problem rate is now negligible.
} 
or normalizing the spectra, and stacking the spectra into the final composite. Each of these steps can have many variations, and their effect on the resulting spectrum can be significant (see Francis et al. 1991, for a discussion of some of these effects). The selection of the input spectra was described in the previous section, and here we detail the remaining steps.

The appropriate statistical methods used to combine the spectra depend upon the spectral quantities of interest. We are interested in both the large-scale continuum shape and the emission-line features of the combined quasars. We have used combining techniques to generate two composite spectra: (1) the median spectrum, which preserves the relative fluxes of the emission features; and (2) the geometric mean spectrum, which preserves the global continuum shape. We have used the geometric mean because quasar continua are often approximated by power laws, and the median (or arithmetic mean) of a sample of power-law spectra will not, in general, result in a power law with the mean index. The geometric mean is defined as, $\left\langle f_{\lambda}\right\rangle_{\mathrm{gm}}=$ $\left(\prod_{i=1}^{n} f_{\lambda, i}\right)^{1 / n}$, where $f_{\lambda, i}$ is the flux density of spectrum number $i$ in the bin centered on wavelength $\lambda$, and $n$ is the number of spectra contributing to the bin. Assuming a power-law form for the continuum flux density, $f_{\lambda} \propto$ $\lambda^{-\left(\alpha_{v}+2\right)}$, it is easily shown that $\left\langle f_{\lambda}\right\rangle_{\mathrm{gm}} \propto \lambda^{-\left(\left\langle\alpha_{v}\right\rangle+2\right)}$, where $\left\langle\alpha_{v}\right\rangle$ is the (arithmetic) mean value of the frequency powerlaw index. [The wavelength index, $\alpha_{\lambda}$, and the frequency index, $\alpha_{v}$, are related by $\left.\alpha_{\lambda}=-\left(\alpha_{v}+2\right)\right]$.

The rest positions of emission lines in quasar spectra, especially the high-ionization broad lines, are known to vary from their nominal laboratory wavelengths (Gaskell 1982; Wilkes 1986; Espey et al. 1989; Zheng \& Sulentic 1990; Corbin 1990; Weymann et al. 1991; Tytler \& Fan 1992; Brotherton et al. 1994b; Laor et al. 1995; McIntosh et al. 1999), so the adopted redshifts of quasars depend upon the lines measured. In addition to understanding the phenomenon of line shifts, unbiased redshifts are important for understanding the nature of associated absorption-line systems (Foltz et al. 1986), for accurately measuring the intergalactic medium ionizing flux (Bajtlik, Duncan, \& Ostriker 1988), and for understanding the dynamics of close pairs of quasars. If the redshifts are consistently measured, say using a common emission line or by cross-correlation with a template, then the mean relative line shifts can be measured accurately with a composite made using those redshifts. For the redshifts of our quasars, we have used only the $[\mathrm{O}$ III $] \lambda 5007$ emission line when possible, since it is narrow, bright, unblended, and is presumed to be emitted at nearly the systemic redshift of the host galaxy (Gaskell 1982; Vrtilek \& Carleton 1985; McIntosh et al. 1999). Some weak Fe II emission is expected near $5000 \AA$ (see, e.g., Wills, Netzer, \& Wills 1985; Verner et al. 1999; Forster et al. 2001), but after subtraction of a local continuum (see $\S 4$ ), contamination of the narrow [O III] line by the broad Fe II complex should be less than a few percent at most. Additionally, we use only the top $\approx 50 \%$ of the emission-line peak to measure its position, which greatly reduces uncertainties due to line asymmetry. An initial composite was made (as described below) using spectra with measured [O III] emission, and this composite was used as a crosscorrelation template for quasars in which the [O III] line was not observable. In this way, all quasars were put onto a common redshift calibration, i.e., relative to the [O III] $\lambda 5007$ line. We now explain this in detail.

\subsection{Generating the $[\mathrm{O} \mathrm{III}]$ Template}

The [O III]-based spectrum was made using 373 spectra with a strong [O III] $\lambda 5007$ emission line unaffected by night-sky lines and includes quasars with redshifts from $z=0.044$ to 0.840 . Spectra were combined at rest wavelengths that were covered by at least three independent spectra, which resulted in a final wavelength coverage of $2070<\lambda<8555 \AA$ for the [O III]-based spectrum. The redshifts were based on the peak position of the [O III] $\lambda 5007$ line, estimated by calculating the mode of the top $\approx 50 \%$ of the line using the relation mode $=3 \times$ median -2 $\times$ mean, which gives better peak estimates than the centroid or median for slightly skewed profiles (see, e.g., Lupton 1993). Uncertainties in the peak positions were estimated by taking into account the errors in the flux density of the pixels contributing to the emission line. The mean uncertainty in the peak positions was $35 \mathrm{~km} \mathrm{~s}^{-1}$ (rest-frame velocity). This is a few times larger than the wavelength calibration uncertainty of less than $10 \mathrm{~km} \mathrm{~s}^{-1}$, based upon spectral observations of radial velocity standards (York et al. 2000). The wavelength array of each spectrum was shifted to the rest frame using the redshift based on the [O III] line. The wavelengths and flux densities were rebinned onto a common dispersion of $1 \AA$ per binroughly the resolution of the observed spectra shifted to the rest frame-while conserving flux. Flux values in pixels that overlapped more than one new bin were distributed among the new bins according to the fraction of the original pixel width covering each new bin. The spectra were ordered by redshift and the flux density of the first spectrum was arbitrarily scaled. The other spectra were scaled in order of redshift to the average of the flux density in the common wavelength region of the mean spectrum of all the lower redshift spectra. The final spectrum was made by finding the median flux density in each bin of the shifted, rebinned, and scaled spectra.

The [O III]-based median composite was then used as a template to refine redshift estimates for those spectra without measurable [O III] emission and those for which the [O III] line was redshifted beyond $9200 \AA$. We used a $\chi^{2}$ minimization technique, similar to that used by Franx, Illingworth, \& Heckman (1989), to measure the redshifts. A low-order polynomial was fitted to the composite and to each spectrum to approximate a continuum and then subtracted. The composite spectrum was shifted in small redshift steps and compared with the individual quasar spectra. The redshift that minimized $\chi^{2}$ - the sum of the squared inverse-variance weighted residuals - was taken as the systemic redshift. Quasars with $\chi^{2}$ and manual redshifts that differed by more than twice the dispersion of the velocity differences of the entire sample (about $700 \mathrm{~km} \mathrm{~s}^{-1}$ ) were examined for possible causes unrelated to the properties of the quasar. Spectra with identified problems were either corrected (if possible) or rejected. A new composite was then made using all the spectra with either $\chi^{2}$ or [O III] redshifts. The template matching and recombining process was done in several progressively higher redshift ranges, so that there was sufficient overlap between the templates and the input spectra that included at least two strong emission lines.

\subsection{Generating the Composite Spectra}

Both median and geometric mean composite spectra were then generated for the analysis of emission features and the global continuum, respectively. The final set of 


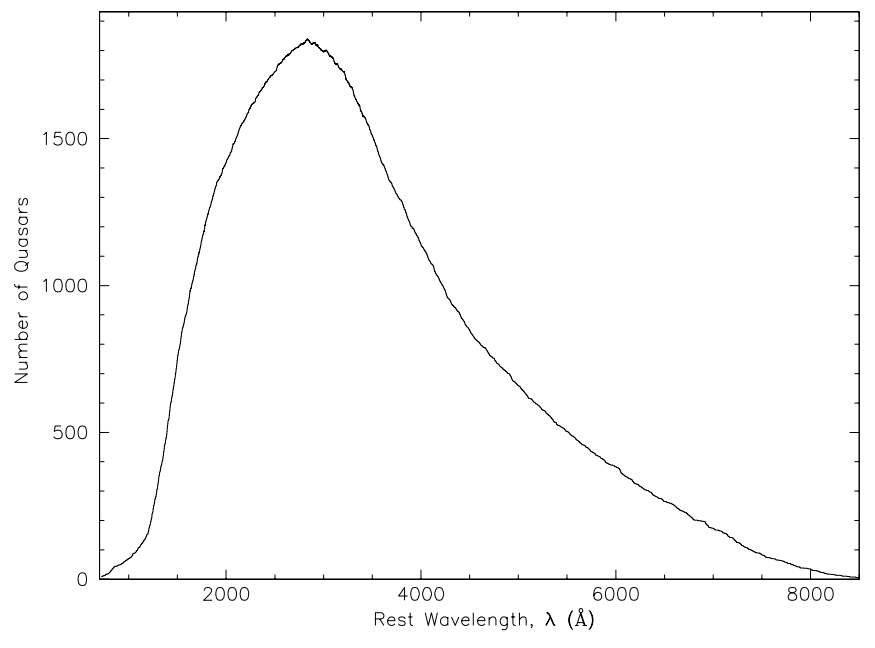

FIG. 2.-Number of quasar spectra combined in each $1 \AA$ bin of the composite as a function of rest wavelength.

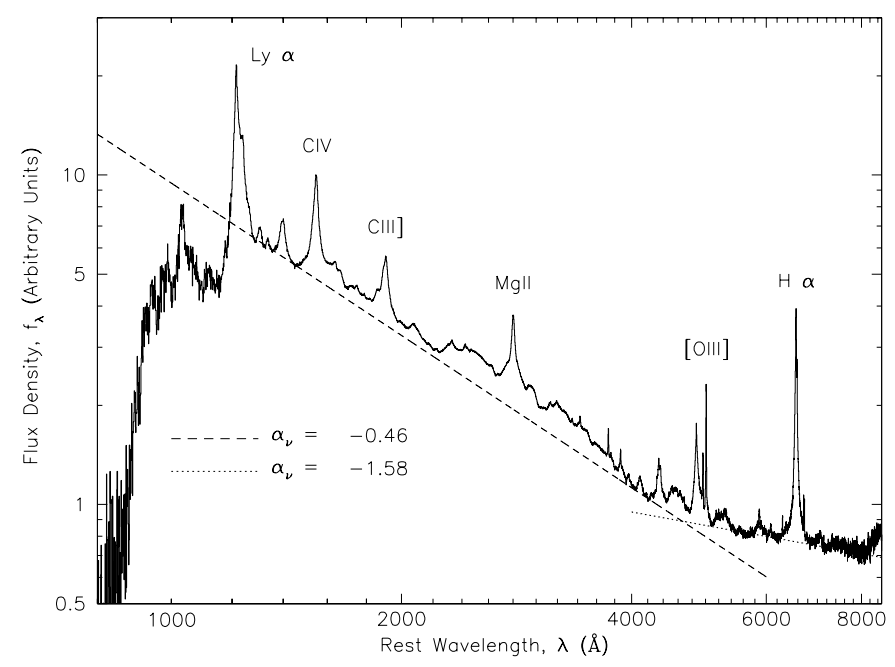

FIG. 3.-Composite quasar spectrum using median combining. Powerlaw fits to the estimated continuum flux are shown. The resolution of the input spectra is $\approx 1800$, which gives a wavelength resolution of about $1 \AA$ in the rest frame.

spectra were shifted to the rest frame using the refined redshifts, then rebinned onto a common wavelength scale at $1 \AA$ per bin, which is roughly the resolution of the observed spectra shifted to the rest frame. The number of quasar spectra that contribute to each $1 \AA$ bin is shown as a function of wavelength in Figure 2. The median spectrum was constructed from the entire data set in the same way as the [O III] composite, as described in the previous section. The spectral region blueward of the Ly $\alpha$ emission line was ignored when calculating the flux density scaling, since the Ly $\alpha$ forest flux density varies greatly from spectrum to spectrum. The final spectrum was truncated to $800 \AA$ on the short-wavelength end, since there was little or no usable flux in the contributing spectra at shorter wavelengths. The median flux density values of the shifted, rebinned, and scaled spectra were determined for each wavelength bin to form the final median composite quasar spectrum, shown in Figure 3 on a logarithmic scale. An error array was calcu-

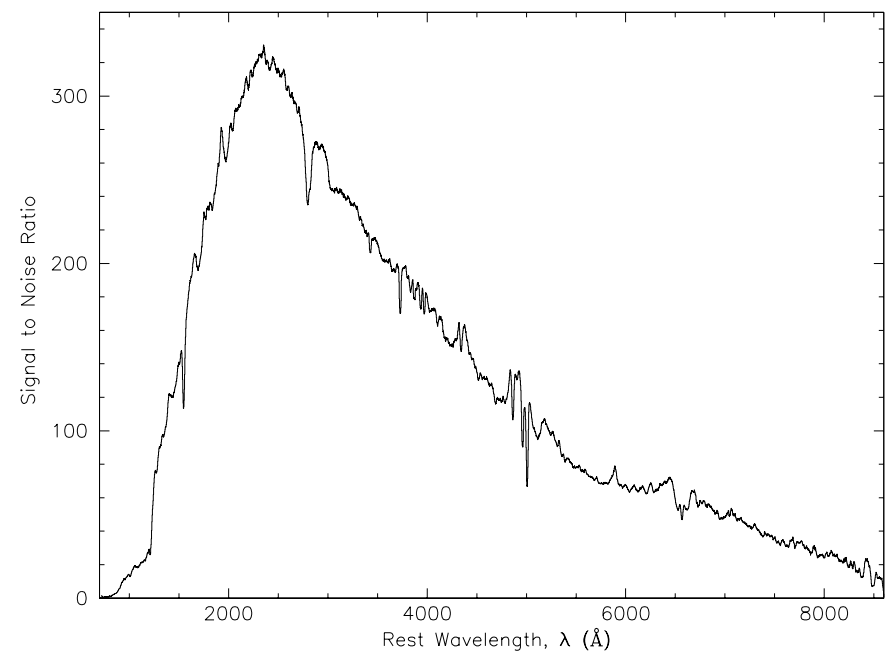

FIG. 4.- S/N per $1 \AA$ bin for the median composite quasar spectrum. The peak reaches almost 330 at $2800 \AA$.

lated by dividing the $68 \%$ semi-interquantile range of the flux densities by the square root of the number of spectra contributing to each bin. This estimate agrees well with the uncertainty determined by measuring the variance in relatively featureless sections of the combined spectrum. The median spectrum extends from 800 to $8555 \AA$ in the rest frame. Figure 4 shows the $\mathrm{S} / \mathrm{N}$ per $1 \AA$ bin, which approaches 330 at $2800 \AA$. The wavelength, flux density, and uncertainty arrays of the median spectrum are given in Table 1.

To generate the geometric mean spectrum, the shifted and rebinned spectra were normalized to unit average flux density over the rest-wavelength interval 3020-3100 ̊, which contains no strong narrow emission lines and is covered by about $90 \%$ of the spectra. The restriction that the input spectra cover this interval results in a combined spectrum that ranges from about 1300 to $7300 \AA$ and is composed of spectra with redshifts from $z=0.26$ to 1.92 .

TABLE 1

Median Composite Quasar Spectrum

\begin{tabular}{ccc}
\hline \hline $\begin{array}{c}\lambda \\
(\AA)\end{array}$ & $\begin{array}{c}f_{\lambda} \\
\text { (arbitrary units) }\end{array}$ & $\begin{array}{c}f_{\lambda} \text { Uncertainty } \\
\text { (arbitrary units) }\end{array}$ \\
\hline $800.5 \ldots \ldots$ & 0.149 & 0.074 \\
$801.5 \ldots \ldots$ & 0.000 & 0.260 \\
$802.5 \ldots \ldots$ & 0.676 & 0.227 \\
$803.5 \ldots \ldots$ & 0.000 & 0.222 \\
$804.5 \ldots \ldots$ & 0.413 & 0.159 \\
$805.5 \ldots \ldots$ & 0.338 & 0.326 \\
$806.5 \ldots \ldots$ & 0.224 & 0.159 \\
$807.5 \ldots \ldots$ & 0.122 & 0.360 \\
$808.5 \ldots \ldots$ & 0.612 & 0.346 \\
$809.5 \ldots \ldots$ & 0.752 & 0.304 \\
$810.5 \ldots \ldots$ & 0.197 & 0.257 \\
$811.5 \ldots \ldots$ & 0.187 & 0.189 \\
$812.5 \ldots \ldots$ & 0.000 & 0.126 \\
$813.5 \ldots \ldots$ & 0.000 & 0.171 \\
$814.5 \ldots \ldots$ & 0.502 & 0.181 \\
\hline
\end{tabular}

Notes.-Table 1 is available in its entirety in the electronic edition of the Astronomical Journal. A portion is shown here for guidance regarding its form and content. 


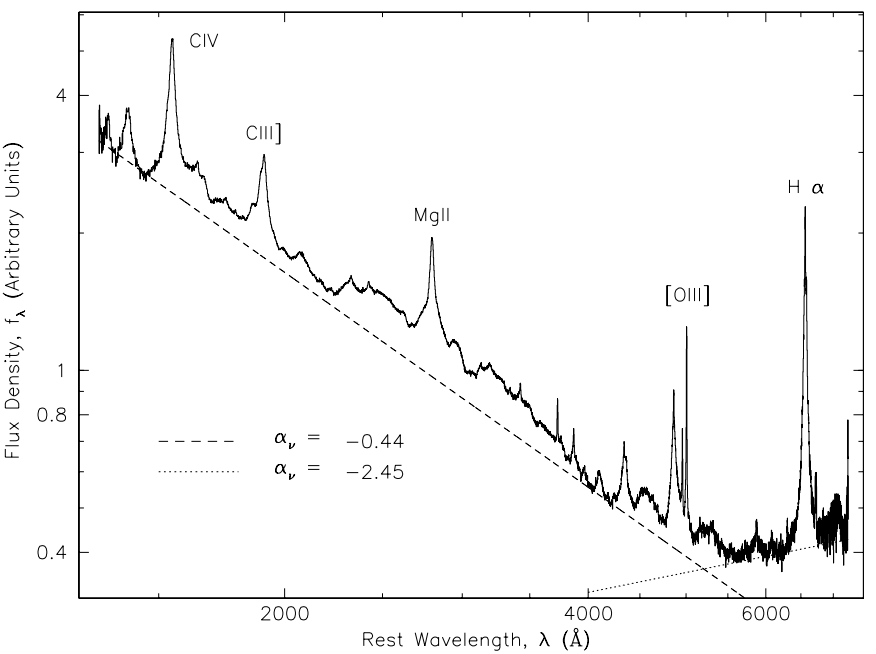

FIG. 5.-Composite quasar spectrum generated using the geometric mean of the input spectra. Power-law fits to the estimated continuum flux are shown. The geometric mean is a better estimator than the arithmetic mean (or median) for power-law distributions. The resolution of the input spectra is $\approx 1800$ in the observed frame, which gives a wavelength resolution of about $1 \AA$ in the rest frame.

The geometric mean of the flux density values was calculated in each wavelength bin to form the geometric mean composite quasar spectrum, shown in Figure 5 on a logarithmic scale. The median and geometric mean composites are quite similar, but there are subtle differences in both the continuum slopes and the emission-line profiles, discussed further in the following sections, which justify the construction of both composite spectra.

\section{CONTINUUM, EMISSION, AND ABSORPTION FEATURES}

\subsection{The Continuum}

The geometric mean spectrum is shown on a log-log scale in Figure 5, in which a single power law will appear as a straight line. The problem of fitting the quasar continuum is complicated by the fact that there are essentially no emission-line-free regions in the spectrum. Our approach is to find a set of regions that give the longest wavelength range over which a power-law fit does not cross the spectrum (i.e., the end points of the fit are defined by the two most widely separated consecutive intersections). The regions that satisfy this are $1350-1365$ and $4200-4230 \AA$. A single power-law fit through the points in those regions gives an index of $\alpha_{v}=-0.44\left(\alpha_{\lambda}=-1.56\right)$ and fits the spectrum reasonably well from just redward of $\mathrm{Ly} \alpha$ to just blueward of $\mathrm{H} \beta$ (Fig. 5). The statistical uncertainty in the spectral index from the fit alone is quite small $(\approx 0.005)$ owing to the high $\mathrm{S} / \mathrm{N}$ of the spectrum and the wide separation of the fitted regions. However, the value of the index is sensitive to the precise wavelength regions used for fitting. More importantly, the spectrophotometric calibration of the spectra introduces an uncertainty of $\approx 0.1$ in $\alpha_{v}(\S 2)$. We estimate the uncertainty of the measured value of the average continuum index to be $\approx 0.1$, based mainly on the remaining spectral response uncertainties. Redward of $\mathrm{H} \beta$ the continuum flux density rises above the amount predict- ed by the power law; this region is better fitted by a separate power law with an index of $\alpha_{v}=-2.45\left(\alpha_{\lambda}=0.45\right.$; Fig. 5), which was determined using the wavelength ranges $6005-$ $6035 \AA$ and $7160-7180 \AA$. The abrupt change in the continuum slope is discussed in $\S 5$.

As a comparison, we have also measured the power-law indices for the median composite, which are $\alpha_{v}=-0.46$ $\left(\alpha_{\lambda}=-1.54\right)$ and $\alpha_{v}=-1.58\left(\alpha_{\lambda}=-0.42\right)$ for the respective wavelength regions (Fig. 3). The index found for the $\mathrm{Ly} \alpha$-to- $\mathrm{H} \beta$ region is almost indistinguishable from that found for the geometric mean composite. The indices for both spectra redward of $\mathrm{H} \beta$ are significantly different, however, and are a result of the different combining processes. The geometric mean should give a better estimate of the average index, but comparison with mean or median composite spectra from other studies is probably reasonable in the $\mathrm{Ly} \alpha$-to- $\mathrm{H} \beta$ region, given the small difference in the indices measured for our composite spectra. The continuum blueward of the Ly $\alpha$ emission line is heavily absorbed by $\operatorname{Ly} \alpha$ forest absorption, as seen in Figure 3. However, because the strength of the $L y \alpha$ forest is a strong function of redshift and a large range of redshifts was used in constructing the sample, no conclusions can be drawn about the absorption or the continuum in that region.

\subsection{Emission and Absorption Lines}

The high $\mathrm{S} / \mathrm{N}$ and relatively high resolution $(1 \AA)$ of the composite allows us to locate and identify weak emission features and resolve some lines that are often blended in other composites. It is also possible that our sample includes a higher fraction of spectra with narrower line profiles, which could also help in distinguishing emission features. For example, close lines that are clearly distinguished in the spectrum include $\mathrm{H} \alpha /[\mathrm{N}$ II $](\lambda \lambda 6548,6563,6583)$, $\mathrm{Si}$ III $] / \mathrm{C}$ III] $(\lambda \lambda 1892,1908)$, the $[\mathrm{S} \mathrm{II}](\lambda \lambda 6716,6730)$ doublet, and $\mathrm{H} \gamma /[\mathrm{O} \mathrm{III}](\lambda \lambda 4340,4363)$. Emission-line features above the continuum were identified manually in the median spectrum. Including the broad Fe II and Fe III complexes, 85 emission features were detected. The endpoints of line positions $\lambda_{\mathrm{lo}}$ and $\lambda_{\mathrm{hi}}$ were estimated to be where the flux density was indistinguishable from the local "continuum." The local continuum is not necessarily the same as the powerlaw continuum estimated in $\S 4.1$, since the emission lines may appear to lie on top of other emission lines or broad Fe II emission features. The peak position of each emission line, $\lambda_{\text {obs }}$, was estimated by calculating the mode of the top $\approx 50 \%$ of the line - the same method used for measuring the [O III] $\lambda 5007$ line peaks in $\S 3.1$. Uncertainties in the peak positions include the contribution from the flux density uncertainties, but none from uncertainties in the local continuum estimate. Fluxes and equivalent widths were measured by integrating the line flux density between the endpoints and above the estimated local continuum. Line profile widths were estimated by measuring the rms wavelength dispersion, $\sigma_{\lambda}$, about the peak position-i.e., the square root of the average flux-weighted squared differences between the wavelength of each pixel in a line profile and the peak line position. Asymmetry of the line profiles was measured using Pearson's skewness coefficient, skewness = $3 \times($ mean - median $) / \sigma_{\lambda}$. Lines were identified by matching wavelength positions and relative strengths of emission features found in other objects, namely the Francis et al. (1991) composite, the Zheng et al. (1997) composite, the narrow-lined quasar I Zw 1 (Laor et al. 1997; Oke \& Lauer 


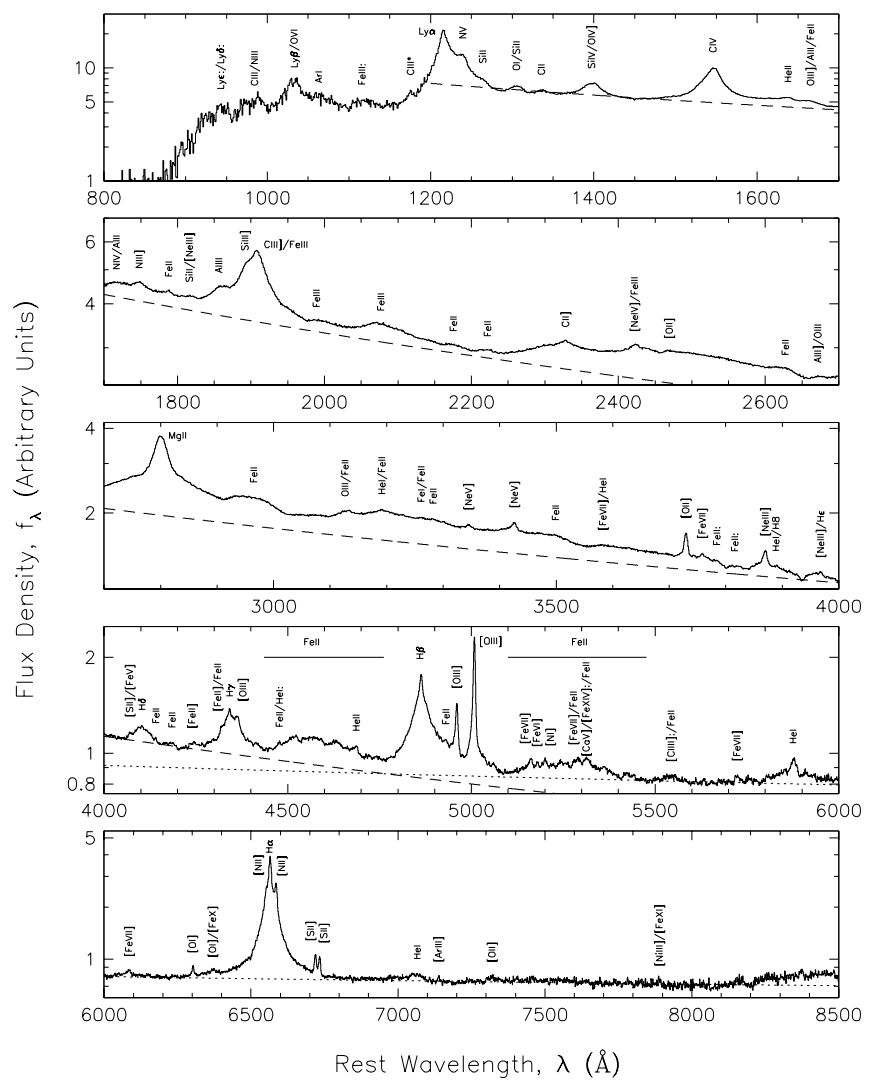

FIG. 6.-Expanded view of median quasar composite on loglinear scale with emission features labeled by ion. Labels ending with a colon (:) are uncertain identifications. The two power-law continuum fits are shown by dashed and dotted lines. The flux from 1600 to $3800 \AA$ is also composed of heavily blended $\mathrm{Fe}$ II and $\mathrm{Fe}$ III lines and Balmer continuum emission.

1979; Phillips 1976), the ultrastrong Fe II emitting quasar 2226-3905 (Graham, Clowes, \& Campusano 1996), the bright Seyfert 1 galaxy NGC 7469 (Kriss et al. 2000), the high-ionization Seyfert 1 galaxy III Zw 77 (Osterbrock 1981), the extensively observed Seyfert 2 galaxy NGC 1068 (Snijders, Netzer, \& Boksenberg 1986), the powerful radio galaxy Cygnus A (Tadhunter, Metz, \& Robinson 1994), and the Orion Nebula H II region (Osterbrock, Tran, \& Veilleux 1992). Identification of many Fe II complexes was made by comparison with predicted multiplet strengths by Verner et al. (1999), Netzer \& Wills (1983), Grandi (1981), and Phillips (1978), and multiplet designations are taken from those references. Table 2 lists the detected lines, their vacuum wavelength peak positions, relative fluxes, equivalent widths, profile widths, skewness, and identifications. Rest wavelengths were taken from the Atomic Line List. ${ }^{29}$ Wavelengths of lines consisting of multiple transitions were found by taking the oscillator-strength-weighted average in the case of permitted lines and the adopted values from the above references for forbidden lines. In all cases, the permitted rest-wavelength values agreed with the (vacuum) values adopted in the above references. Figure 6 shows an expanded view of the quasar composite on a loglinear scale with the emission features labeled.

\footnotetext{
${ }^{29}$ The Atomic Line List is hosted by the Department of Physics and Astronomy at the University of Kentucky (see http://www.pa.uky.edu/ $\sim$ peter/atomic/).
}

It is clear from Figure 6 that most of the UV-optical continuum is populated by emission lines. Most strong emission lines show "contamination" by blends with weaker lines, as seen in the expanded profiles of 12 emission-line regions in Figure 7. The very broad conspicuous feature from $\approx 2200$ to $4000 \AA$ is known as the $3000 \AA$ bump (Grandi 1982; Oke, Shields, \& Korycansky 1984) and consists of blends of $\mathrm{Fe}$ II line emission and Balmer continuum emission (Wills et al. 1985). The Fe II and Fe III complexes are particularly ubiquitous and contribute a large fraction of the emission-line flux. Using this composite, these complexes have been shown to be an important contributor to the color-redshift relationships of quasars (Richards et al. 2001b).

Several absorption features often seen in galaxies are also identifiable in the median composite quasar spectrum. These lines are listed in Table 3, along with several measured quantities, and include H9 $\lambda 3835, \mathrm{H} 10$ $\lambda 3797$, the $\mathrm{Ca}$ II $\lambda 3933 \mathrm{~K}$ line, and Ca II $\lambda \lambda 8498,8542$ - two lines of a triplet (the second weakest third component would fall beyond the red end of the spectrum). The $\mathrm{Mg}$ I $\lambda \lambda 5167$, 5172,5183 triplet lines may also be present in the spectrum, but they would lie inside a strong complex of Fe II emission and near several other expected emission lines. The locations of other common stellar absorption lines seen in galaxies, such as the lower order Balmer lines and the $\mathrm{Ca}$ II $\lambda 3968 \mathrm{H}$ line, are dominated by emission lines. The presence of stellar absorption lines argues for at least some hostgalaxy contamination in the quasar composite spectrum, despite the fact that we rejected objects with obvious stellar lines in individual galaxies. To examine this further, we have created a low-redshift median composite using only quasars with redshifts of $z_{\mathrm{em}} \leq 0.5$, which is almost equivalent to selecting only quasars with rest-frame absolute $r^{\prime}$ magnitude $M_{r^{\prime}} \geq-21.5$ (calculated using a spectral index of $\alpha_{v}=$ $-0.44)$. The low- $z$ composite covers a rest-wavelength range of $2550-8555 \AA$. The absorption lines found in the low- $z$ spectrum are marked in Figure 8 and listed in Table 3. More absorption lines are detected in the low- $z$ composite spectrum than the full data set spectrum, and the lines in common are stronger in the low- $z$ spectrum - as expected if host-galaxy contamination is the source of the absorption lines. We discuss the absorption lines in more detail in $\S 5$.

The $2175 \AA$ extinction bump often seen in the spectra of objects observed through the Galactic diffuse interstellar medium and usually attributed to graphite grains (Mathis, Rumpl, \& Nordsieck 1977) is not present at a detectable level in the composite spectrum. This agrees with the nondetection of the feature by Pitman, Clayton, \& Gordon (2000) who searched for it in other quasar spectral composites.

\subsection{Systematic Line Shifts}

Because the composite was constructed using redshifts based on a single emission-line position ([O III] 25007$)$ or on cross-correlations with an [O III]-based composite, we can check for systematic offsets between the measured peak positions and the [O III]-based wavelengths. Several emission lines - C IV $\lambda 1549$, for example - are offset from their laboratory wavelengths, as evident from Figure 7. Such line shifts have been detected previously (see, e.g., Grandi 1982; Wilkes 1986; Tytler \& Fan 1992; Laor et al. 1995; McIntosh et al. 1999) and are present for many of the lines listed in Table 2. Real line position offsets can be confused with 
TABLE 2

Composite Quasar Emission-Line Features

\begin{tabular}{|c|c|c|c|c|c|c|c|c|}
\hline $\begin{array}{l}\lambda_{\text {obs }} \\
(\AA)\end{array}$ & $\begin{array}{l}\lambda_{\mathrm{lo}} \\
(\AA)\end{array}$ & $\begin{array}{l}\lambda_{\mathrm{hi}} \\
(\AA \AA)\end{array}$ & $\begin{array}{c}\text { Rel. Flux } \\
{[100 \times F / F(\operatorname{Ly} \alpha)]}\end{array}$ & $\begin{array}{l}W \\
(\AA)\end{array}$ & $\begin{array}{l}\text { Width } \sigma_{\lambda} \\
(\AA)\end{array}$ & Skew & $\mathrm{ID}^{\mathrm{a}}$ & $\lambda_{\text {lab }}$ or Multiplet ${ }_{(\AA)}^{\mathrm{b}}$ \\
\hline \multirow[t]{2}{*}{$940.93 \pm 3.17 \ldots \ldots}$. & 930 & 955 & $2.110 \pm 0.379$ & $2.95 \pm 0.54$ & 4.73 & 0.59 & Ly $\epsilon:$ & 937.80 \\
\hline & $\ldots$ & $\ldots$ & $\ldots$ & $\ldots$ & $\ldots$ & $\ldots$ & Ly $\delta:$ & 949.74 \\
\hline \multirow[t]{2}{*}{$985.46 \pm 4.78 \ldots \ldots}$. & 960 & 1003 & $5.195 \pm 0.459$ & $6.55 \pm 0.58$ & 8.95 & -0.51 & $\mathrm{C}$ III & 977.02 \\
\hline & $\ldots$ & $\ldots$ & $\ldots$ & $\ldots$ & $\ldots$ & $\ldots$ & $\mathrm{N}$ III & 990.69 \\
\hline \multirow[t]{2}{*}{$1033.03 \pm 1.27 \ldots \ldots$} & 1012 & 1055 & $9.615 \pm 0.484$ & $9.77 \pm 0.49$ & 7.76 & -0.01 & $\operatorname{Ly} \beta$ & 1025.72 \\
\hline & $\ldots$ & $\ldots$ & $\ldots$ & $\ldots$ & $\ldots$ & $\ldots$ & $\mathrm{O}_{\mathrm{VI}}$ & 1033.83 \\
\hline $1065.10 \pm 5.09 \ldots \ldots$ & 1055 & 1077 & $0.816 \pm 0.269$ & $0.80 \pm 0.27$ & 4.17 & 0.30 & Ar I & 1066.66 \\
\hline $1117.26 \pm 2.78 \ldots \ldots$ & 1100 & 1140 & $3.151 \pm 0.289$ & $3.66 \pm 0.34$ & 8.49 & 0.02 & Fe III: & UV 1 \\
\hline $1175.35 \pm 1.17 \ldots \ldots$ & 1170 & 1182 & $0.870 \pm 0.148$ & $0.83 \pm 0.14$ & 2.28 & -0.01 & $\mathrm{C}$ III* & 1175.70 \\
\hline $1216.25 \pm 0.37 \ldots \ldots$ & 1160 & 1290 & $100.000 \pm 0.753$ & $92.91 \pm 0.72$ & 19.46 & 0.40 & $\operatorname{Ly} \alpha$ & 1215.67 \\
\hline $1239.85 \pm 0.67 \ldots \ldots$ & 1230 & 1252 & $2.461 \pm 0.189$ & $1.11 \pm 0.09$ & 2.71 & -0.21 & $\mathrm{~N} v$ & 1240.14 \\
\hline $1265.22 \pm 3.20 \ldots \ldots$ & 1257 & 1274 & $0.306 \pm 0.081$ & $0.21 \pm 0.06$ & 2.74 & 0.25 & Si II & 1262.59 \\
\hline \multirow{2}{*}{$1305.42 \pm 0.71 \ldots \ldots$} & 1290 & 1318 & $1.992 \pm 0.076$ & $1.66 \pm 0.06$ & 5.42 & -0.21 & $\mathrm{O}_{\mathrm{I}}$ & 1304.35 \\
\hline & $\ldots$ & $\cdots$ & $\ldots$ & $\ldots$ & $\ldots$ & $\ldots$ & Si II & 1306.82 \\
\hline $1336.60 \pm 1.13 \ldots \ldots$ & 1325 & 1348 & $0.688 \pm 0.059$ & $0.59 \pm 0.05$ & 3.86 & -0.02 & $\mathrm{C}_{\text {III }}$ & 1335.30 \\
\hline \multirow[t]{2}{*}{$1398.33 \pm 0.31 \ldots \ldots$} & 1360 & 1446 & $8.916 \pm 0.097$ & $8.13 \pm 0.09$ & 12.50 & 0.06 & Si IV & 1396.76 \\
\hline & $\ldots$ & $\ldots$ & $\ldots$ & $\ldots$ & $\ldots$ & $\ldots$ & O Iv] & 1402.06 \\
\hline $1546.15 \pm 0.14 \ldots \ldots$ & 1494 & 1620 & $25.291 \pm 0.106$ & $23.78 \pm 0.10$ & 14.33 & -0.04 & $\mathrm{C}$ IV & 1549.06 \\
\hline $1637.84 \pm 0.83 \ldots \ldots$ & 1622 & 1648 & $0.521 \pm 0.027$ & $0.51 \pm 0.03$ & 4.43 & -0.22 & He II & 1640.42 \\
\hline \multirow[t]{3}{*}{$1664.74 \pm 1.04 \ldots \ldots$} & 1648 & 1682 & $0.480 \pm 0.028$ & $0.50 \pm 0.03$ & 5.50 & 0.11 & $\mathrm{O} \mathrm{III}]$ & 1663.48 \\
\hline & $\ldots$ & $\ldots$ & $\ldots$ & $\ldots$ & $\ldots$ & $\ldots$ & $\mathrm{Al}$ II & 1670.79 \\
\hline & $\ldots$ & $\ldots$ & $\ldots$ & $\ldots$ & $\ldots$ & $\ldots$ & $\mathrm{Fe}$ II & UV 40 \\
\hline \multirow[t]{3}{*}{$1716.88 \pm 2.83 \ldots \ldots$} & 1696 & 1736 & $0.258 \pm 0.027$ & $0.30 \pm 0.03$ & 7.36 & 0.17 & $\mathrm{~N}$ IV & 1718.55 \\
\hline & $\ldots$ & $\ldots$ & $\ldots$ & .. & $\ldots$ & $\ldots$ & $\mathrm{Fe}$ II & UV 37 \\
\hline & $\cdots$ & $\cdots$ & $\ldots$ & $\ldots$ & $\cdots$ & $\cdots$ & $\mathrm{Al}$ II & 1721.89 \\
\hline $1748.31 \pm 0.75 \ldots \ldots$ & 1735 & 1765 & $0.382 \pm 0.021$ & $0.44 \pm 0.03$ & 5.12 & 0.04 & $\mathrm{~N}$ iII] & 1750.26 \\
\hline $1788.73 \pm 0.98 \ldots \ldots$ & 1771 & 1802 & $0.229 \pm 0.020$ & $0.28 \pm 0.02$ & 6.06 & -0.29 & $\mathrm{Fe}$ II & UV 191 \\
\hline \multirow[t]{2}{*}{$1818.17 \pm 2.07 \ldots \ldots$} & 1802 & 1831 & $0.130 \pm 0.019$ & $0.16 \pm 0.02$ & 5.72 & -0.47 & Si II & 1816.98 \\
\hline & $\ldots$ & $\ldots$ & $\ldots$ & $\ldots$ & $\ldots$ & $\ldots$ & {$[\mathrm{Ne}$ III $]$} & 1814.73 \\
\hline $1856.76 \pm 1.18 \ldots \ldots$ & 1840 & 1875 & $0.333 \pm 0.021$ & $0.40 \pm 0.03$ & 4.95 & 0.01 & $\mathrm{Al}$ III & 1857.40 \\
\hline \multirow[t]{2}{*}{$1892.64 \pm 0.83 \ldots \ldots$} & 1884 & 1900 & $0.158 \pm 0.015$ & $0.16 \pm 0.02$ & 3.09 & -0.10 & $\mathrm{Si} \mathrm{III}]$ & 1892.03 \\
\hline & $\ldots$ & $\ldots$ & $\ldots$ & $\ldots$ & $\ldots$ & $\ldots$ & $\mathrm{Fe}$ III & UV 34 \\
\hline \multirow[t]{4}{*}{$1905.97 \pm 0.12 \ldots \ldots$} & 1830 & 1976 & $15.943 \pm 0.041$ & $21.19 \pm 0.05$ & 23.58 & -0.27 & $\mathrm{C}$ III] & 1908.73 \\
\hline & $\ldots$ & $\ldots$ & $\ldots$ & $\ldots$ & $\ldots$ & $\ldots$ & $\mathrm{Fe}$ III & U34 \\
\hline & $\ldots$ & $\ldots$ & $\ldots$ & $\ldots$ & $\ldots$ & $\ldots$ & $\mathrm{Fe}$ III & UV 68 \\
\hline & $\ldots$ & $\ldots$ & $\ldots$ & $\ldots$ & $\ldots$ & $\ldots$ & $\mathrm{Fe}$ III & UV 61 \\
\hline $1991.83 \pm 2.91 \ldots \ldots$ & 1976 & 2008 & $0.139 \pm 0.014$ & $0.20 \pm 0.02$ & 6.73 & -0.03 & $\mathrm{Fe}$ III & UV 50 \\
\hline $2076.62 \pm 0.78 \ldots \ldots$ & 2036 & 2124 & $1.580 \pm 0.021$ & $2.46 \pm 0.03$ & 16.99 & 0.18 & $\mathrm{Fe}$ III & UV 48 \\
\hline \multirow[t]{2}{*}{$2175.62 \pm 1.83 \ldots \ldots$} & 2153 & 2199 & $0.143 \pm 0.013$ & $0.25 \pm 0.02$ & 5.85 & 0.46 & $\mathrm{Fe}$ II & UV 79 \\
\hline & $\ldots$ & $\ldots$ & $\ldots$ & $\ldots$ & $\ldots$ & $\ldots$ & $\mathrm{Fe}$ II & UV 370 \\
\hline \multirow{2}{*}{$2222.29 \pm 1.44 \ldots \ldots$} & 2202 & 2238 & $0.185 \pm 0.011$ & $0.33 \pm 0.02$ & 6.98 & -0.11 & $\mathrm{Fe}$ II & UV 118 \\
\hline & $\ldots$ & $\ldots$ & $\ldots$ & $\ldots$ & $\ldots$ & $\ldots$ & $\mathrm{Fe}$ II & UV 376 \\
\hline $2324.58 \pm 0.56 \ldots \ldots$ & 2257 & 2378 & $2.008 \pm 0.020$ & $3.56 \pm 0.04$ & 22.23 & -0.29 & $\mathrm{Fe}$ II & Many \\
\hline $2327.34 \pm 0.72 \ldots \ldots$ & 2312 & 2338 & $0.183 \pm 0.009$ & $0.31 \pm 0.02$ & 4.95 & -0.41 & $\mathrm{C} \mathrm{II}]$ & 2326.44 \\
\hline $2423.46 \pm 0.44 \ldots \ldots$ & 2402 & 2448 & $0.437 \pm 0.012$ & $0.77 \pm 0.02$ & 8.42 & 0.25 & {$[\mathrm{Ne}$ IV $]$} & 2423.83 \\
\hline & $\ldots$ & $\ldots$ & $\ldots$ & $\ldots$ & $\ldots$ & $\ldots$ & $\mathrm{Fe}$ III & UV 47 \\
\hline $2467.98 \pm 1.59 \ldots \ldots$ & 2458 & 2482 & $0.092 \pm 0.009$ & $0.16 \pm 0.02$ & 4.54 & 0.30 & {$[\mathrm{O}$ II $]$} & 2471.03 \\
\hline & $\ldots$ & 2. & $\ldots$ & 0.0010 .04 & 100 & 0.00 & $\mathrm{Fe}$ II & UV 395 \\
\hline $2626.92 \pm 0.99 \ldots \ldots$ & 2595 & 2654 & $0.398 \pm 0.013$ & $0.81 \pm 0.03$ & 9.93 & 0.00 & $\mathrm{Fe}$ II & UV 1 \\
\hline $2671.89 \pm 1.78 \ldots \ldots$ & 2657 & 2684 & $0.067 \pm 0.008$ & $0.14 \pm 0.02$ & 5.10 & 0.05 & $\mathrm{Al} \mathrm{II}]$ & 2669.95 \\
\hline & $\ldots$ & $\ldots$ & -5 & 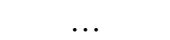 & 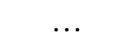 & $\ldots$ & $\mathrm{O}$ III & 2672.04 \\
\hline $2800.26 \pm 0.10 \ldots \ldots$ & 2686 & 2913 & $14.725 \pm 0.030$ & $32.28 \pm 0.07$ & 34.95 & -0.06 & Mg II & 2798.75 \\
\hline $2964.28 \pm 0.79 \ldots \ldots$ & 2910 & 3021 & $2.017 \pm 0.017$ & $4.93 \pm 0.04$ & 22.92 & -0.03 & $\mathrm{Fe}$ II & UV 78 \\
\hline $3127.70 \pm 1.07 \ldots \ldots$ & 3100 & 3153 & $0.326 \pm 0.012$ & $0.86 \pm 0.03$ & 9.38 & -0.13 & O III & 3133.70 \\
\hline & $\ldots$ & $\ldots$ & $\cdots$ & $\ldots$ & $\ldots$ & $\ldots$ & $\mathrm{Fe}$ II & Opt 82 \\
\hline $3191.78 \pm 0.99 \ldots \ldots$ & 3159 & 3224 & $0.445 \pm 0.013$ & $1.17 \pm 0.03$ & 12.77 & -0.04 & He I & 3188.67 \\
\hline & $\ldots$ & $\ldots$ & $\ldots$ & .. & $\ldots$ & $\ldots$ & $\mathrm{Fe}$ II & Opt 6 \\
\hline & $\ldots$ & $\ldots$ & $\ldots$ & $\ldots$ & $\ldots$ & $\ldots$ & $\mathrm{Fe}$ II & Opt 7 \\
\hline $3261.40 \pm 2.70 \ldots \ldots$ & 3248 & 3272 & $0.032 \pm 0.008$ & $0.09 \pm 0.02$ & 3.27 & 0.06 & $\mathrm{Fe}_{\mathrm{I}}$ & Opt 91 \\
\hline & $\ldots$ & 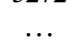 & $\ldots$ & $\ldots$ & ( & $\ldots$ & $\mathrm{Fe}$ II & Opt 1 \\
\hline $3281.74 \pm 3.15 \ldots \ldots$ & 3272 & 3297 & $0.036 \pm 0.008$ & $0.10 \pm 0.02$ & 4.39 & 0.56 & $\mathrm{Fe}$ II & Opt 1 \\
\hline $3345.39 \pm 0.75 \ldots \ldots$ & 3329 & 3356 & $0.118 \pm 0.008$ & $0.35 \pm 0.02$ & 5.50 & -0.41 & {$[\mathrm{Ne} \mathrm{v}]$} & 3346.82 \\
\hline $3425.66 \pm 0.46 \ldots \ldots$ & 3394 & 3446 & $0.405 \pm 0.012$ & $1.22 \pm 0.04$ & 9.09 & -0.62 & {$[\mathrm{Ne} v]$} & 3426.84 \\
\hline $3498.92 \pm 1.60 \ldots \ldots$ & 3451 & 3537 & $0.432 \pm 0.014$ & $1.38 \pm 0.05$ & 16.79 & -0.24 & $\mathrm{Fe}$ II & Opt 4 \\
\hline
\end{tabular}


TABLE 2-Continued

\begin{tabular}{|c|c|c|c|c|c|c|c|c|}
\hline $\begin{array}{l}\lambda_{\text {obs }} \\
(\AA)\end{array}$ & $\begin{array}{l}\lambda_{\mathrm{lo}} \\
(\AA)\end{array}$ & $\begin{array}{l}\lambda_{\mathrm{hi}} \\
(\AA)\end{array}$ & $\begin{array}{c}\text { Rel. Flux } \\
{[100 \times F / F(\operatorname{Ly} \alpha)]}\end{array}$ & $\begin{array}{l}W \\
(\AA)\end{array}$ & $\begin{array}{l}\text { Width } \sigma_{\lambda} \\
(\AA)\end{array}$ & Skew & $\mathrm{ID}^{\mathrm{a}}$ & $\lambda_{\text {lab }}$ or Multiplet ${ }_{(\AA)}^{\mathrm{b}}$ \\
\hline & $\ldots$ & $\ldots$ & $\ldots$ & $\ldots$ & $\ldots$ & $\ldots$ & $\mathrm{Fe}$ II & Opt 16 \\
\hline \multirow[t]{2}{*}{$3581.70 \pm 4.48 \ldots \ldots$} & 3554 & 3613 & $0.100 \pm 0.011$ & $0.34 \pm 0.04$ & 7.98 & 0.79 & {$[\mathrm{Fe}$ VII $]$} & 3587.34 \\
\hline & $\ldots$ & $\ldots$ & 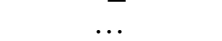 & 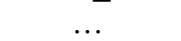 & $\ldots$ & $\ldots$ & $\mathrm{He} \mathrm{I}$ & 3588.30 \\
\hline $3729.66 \pm 0.18 \ldots \ldots$ & 3714 & 3740 & $0.424 \pm 0.009$ & $1.56 \pm 0.03$ & 3.32 & -0.24 & {$[\mathrm{O} \mathrm{II}]$} & 3728.48 \\
\hline $3758.46 \pm 0.56 \ldots \ldots$ & 3748 & 3771 & $0.078 \pm 0.007$ & $0.29 \pm 0.03$ & 3.71 & 0.12 & {$[\mathrm{Fe}$ VII $]$} & 3759.99 \\
\hline $3785.47 \pm 1.31 \ldots \ldots$ & 3775 & 3799 & $0.056 \pm 0.006$ & $0.22 \pm 0.03$ & 4.24 & 0.13 & Fe II: & Opt 15 \\
\hline $3817.41 \pm 2.46 \ldots \ldots$ & 3800 & 3832 & $0.124 \pm 0.007$ & $0.51 \pm 0.03$ & 7.33 & -0.10 & Fe II: & Opt 14 \\
\hline $3869.77 \pm 0.25 \ldots \ldots$ & 3850 & 3884 & $0.345 \pm 0.008$ & $1.38 \pm 0.03$ & 5.31 & -0.50 & {$[\mathrm{Ne}$ III $]$} & 3869.85 \\
\hline \multirow[t]{2}{*}{$3891.03 \pm 1.28 \ldots \ldots$} & 3882 & 3898 & $0.020 \pm 0.005$ & $0.08 \pm 0.02$ & 2.02 & -0.27 & $\mathrm{He} \mathrm{I}$ & 3889.74 \\
\hline & $\ldots$ & $\ldots$ & $\ldots$ & $\ldots$ & $\ldots$ & $\ldots$ & $\mathrm{H} 8$ & 3890.15 \\
\hline \multirow{2}{*}{$3968.43 \pm 0.91 \ldots \ldots$} & 3950 & 3978 & $0.104 \pm 0.007$ & $0.45 \pm 0.03$ & 5.32 & -0.62 & {$[\mathrm{Ne}$ III $]$} & 3968.58 \\
\hline & $\ldots$ & $\ldots$ & $\ldots$ & $\ldots$ & $\ldots$ & $\ldots$ & $\mathrm{H} \epsilon$ & 3971.20 \\
\hline \multirow[t]{2}{*}{$4070.71 \pm 1.18 \ldots \ldots$} & 4061 & 4079 & $0.039 \pm 0.005$ & $0.18 \pm 0.03$ & 3.20 & 0.01 & {$[\mathrm{Fe} \mathrm{v}]$} & 4072.39 \\
\hline & $\ldots$ & $\ldots$ & $\ldots$ & $\ldots$ & $\ldots$ & $\ldots$ & {$[\mathrm{S}$ II $]$} & 4073.63 \\
\hline $4102.73 \pm 0.66 \ldots \ldots$ & 4050 & 4152 & $1.066 \pm 0.013$ & $5.05 \pm 0.06$ & 18.62 & 0.03 & $\mathrm{H} \delta$ & 4102.89 \\
\hline \multirow[t]{2}{*}{$4140.50 \pm 0.96 \ldots \ldots$} & 4135 & 4145 & $0.026 \pm 0.004$ & $0.13 \pm 0.02$ & 1.83 & -0.38 & $\mathrm{Fe}$ II & Opt 27 \\
\hline & $\ldots$ & $\ldots$ & $\ldots$ & $\ldots$ & $\ldots$ & $\ldots$ & $\mathrm{Fe}$ II & Opt 28 \\
\hline \multirow[t]{2}{*}{$4187.55 \pm 1.97 \ldots \ldots$} & 4157 & 4202 & $0.154 \pm 0.009$ & $0.76 \pm 0.04$ & 9.77 & -0.40 & $\mathrm{Fe}$ II & Opt 27 \\
\hline & $\ldots$ & $\ldots$ & $\ldots$ & $\ldots$ & $\ldots$ & $\ldots$ & $\mathrm{Fe}$ II & Opt 28 \\
\hline $4239.85 \pm 2.07 \ldots \ldots$ & 4227 & 4260 & $0.107 \pm 0.008$ & $0.53 \pm 0.04$ & 5.73 & -0.05 & {$[\mathrm{Fe} \mathrm{II}]$} & Opt $21 \mathrm{~F}$ \\
\hline \multirow[t]{2}{*}{$4318.30 \pm 0.78 \ldots \ldots$} & 4315 & 4328 & $0.038 \pm 0.005$ & $0.17 \pm 0.02$ & 2.31 & 0.58 & {$[\mathrm{Fe}$ II $]$} & Opt $21 \mathrm{~F}$ \\
\hline & $\ldots$ & $\ldots$ & $\ldots$ & $\ldots$ & $\ldots$ & $\ldots$ & $\mathrm{Fe}$ II & Opt 32 \\
\hline $4346.42 \pm 0.38 \ldots \ldots$ & 4285 & 4412 & $2.616 \pm 0.017$ & $12.62 \pm 0.08$ & 20.32 & 0.12 & $\mathrm{H} \gamma$ & 4341.68 \\
\hline $4363.85 \pm 0.68 \ldots \ldots$ & 4352 & 4372 & $0.110 \pm 0.007$ & $0.46 \pm 0.03$ & 3.10 & -0.18 & {$[\mathrm{O}$ III $]$} & 4364.44 \\
\hline \multirow{2}{*}{$4478.22 \pm 1.13 \ldots \ldots$} & 4469 & 4484 & $0.029 \pm 0.006$ & $0.14 \pm 0.03$ & 2.20 & -0.69 & $\mathrm{Fe}$ II & Opt 37 \\
\hline & $\ldots$ & $\ldots$ & $\ldots$ & $\ldots$ & $\ldots$ & $\ldots$ & He I: & 4472.76 \\
\hline \multirow[t]{2}{*}{$4564.71 \pm 1.56 \ldots \ldots$} & 4435 & 4762 & $3.757 \pm 0.029$ & $19.52 \pm 0.15$ & $61.69^{c}$ & 0.23 & $\mathrm{Fe}$ II & Opt 37 \\
\hline & $\ldots$ & $\ldots$ & $\ldots$ & $\ldots$ & $\ldots$ & $\ldots$ & $\mathrm{Fe}$ II & Opt 38 \\
\hline $4686.66 \pm 1.04 \ldots \ldots$ & 4668 & 4696 & $0.139 \pm 0.009$ & $0.72 \pm 0.05$ & 5.92 & -0.57 & He II & 4687.02 \\
\hline $4853.13 \pm 0.41 \ldots \ldots$ & 4760 & 4980 & $8.649 \pm 0.030$ & $46.21 \pm 0.16$ & 40.44 & 0.61 & $\mathrm{H} \beta$ & 4862.68 \\
\hline $4930.75 \pm 1.13 \ldots \ldots$ & 4920 & 4941 & $0.082 \pm 0.007$ & $0.40 \pm 0.04$ & 3.98 & -0.02 & $\mathrm{Fe}$ II & Opt 42 \\
\hline $4960.36 \pm 0.22 \ldots \ldots$ & 4945 & 4972 & $0.686 \pm 0.014$ & $3.50 \pm 0.07$ & 3.85 & -0.22 & [O III $]$ & 4960.30 \\
\hline $5008.22 \pm 0.17 \ldots \ldots$ & 4982 & 5035 & $2.490 \pm 0.031$ & $13.23 \pm 0.16$ & 6.04 & -0.22 & {$[\mathrm{O} \mathrm{III}]$} & 5008.24 \\
\hline \multirow[t]{2}{*}{$5305.97 \pm 1.99 \ldots \ldots$} & 5100 & 5477 & $3.522 \pm 0.036$ & $21.47 \pm 0.22$ & $74.83^{\mathrm{c}}$ & -0.25 & $\mathrm{Fe}$ II & Opt 48 \\
\hline & $\ldots$ & $\ldots$ & $\ldots$ & $\ldots$ & $\ldots$ & $\ldots$ & $\mathrm{Fe}$ II & Opt 49 \\
\hline $5160.81 \pm 0.94 \ldots \ldots$ & 5149 & 5168 & $0.092 \pm 0.008$ & $0.52 \pm 0.04$ & 3.95 & -0.42 & {$[\mathrm{Fe} \mathrm{VII}]$} & 5160.33 \\
\hline $5178.68 \pm 0.93 \ldots \ldots$ & 5170 & 5187 & $0.056 \pm 0.007$ & $0.32 \pm 0.04$ & 2.97 & -0.23 & {$[\mathrm{Fe} \mathrm{VI}]$} & 5177.48 \\
\hline $5201.06 \pm 0.98 \ldots \ldots$ & 5187 & 5211 & $0.098 \pm 0.008$ & $0.56 \pm 0.05$ & 4.42 & -0.37 & {$\left[\mathrm{~N}_{\mathrm{I}}\right]$} & 5200.53 \\
\hline \multirow[t]{2}{*}{$5277.92 \pm 2.96 \ldots \ldots$} & 5273 & 5287 & $0.025 \pm 0.007$ & $0.14 \pm 0.04$ & 2.84 & 0.38 & {$[\mathrm{Fe} \mathrm{VII}]$} & 5277.85 \\
\hline & $\ldots$ & $\ldots$ & $\ldots$ & $\ldots$ & $\ldots$ & $\ldots$ & $\mathrm{Fe}$ II & Opt 49 \\
\hline \multirow[t]{4}{*}{$5313.82 \pm 1.85 \ldots \ldots$} & 5302 & 5328 & $0.118 \pm 0.010$ & $0.66 \pm 0.06$ & 5.34 & 0.18 & {$[\mathrm{Fe}$ XIV $]:$} & 5304.34 \\
\hline & $\ldots$ & $\ldots$ & $\ldots$ & $\ldots$ & $\ldots$ & $\ldots$ & {$[\mathrm{Ca} \mathrm{v}]$} & 5310.59 \\
\hline & $\ldots$ & $\ldots$ & $\ldots$ & $\ldots$ & $\ldots$ & $\ldots$ & $\mathrm{Fe}$ II & Opt 48 \\
\hline & $\ldots$ & $\ldots$ & $\ldots$ & $\ldots$ & $\ldots$ & $\ldots$ & $\mathrm{Fe}$ II & Opt 49 \\
\hline \multirow[t]{2}{*}{$5545.16 \pm 4.03 \ldots \ldots$} & 5490 & 5592 & $0.333 \pm 0.021$ & $2.12 \pm 0.13$ & 21.09 & -0.04 & {$[\mathrm{Cl}$ III $]:$} & 5539.43 \\
\hline & $\ldots$ & $\ldots$ & $\ldots$ & $\ldots$ & $\ldots$ & $\ldots$ & $\mathrm{Fe}$ II & Opt 55 \\
\hline $5723.74 \pm 1.94 \ldots \ldots$ & 5704 & 5745 & $0.109 \pm 0.015$ & $0.70 \pm 0.09$ & 5.19 & 0.72 & {$[\mathrm{Fe}$ VII $]$} & 5722.30 \\
\hline $5877.41 \pm 0.81 \ldots \ldots$ & 5805 & 5956 & $0.798 \pm 0.029$ & $4.94 \pm 0.18$ & 23.45 & 0.26 & $\mathrm{He} \mathrm{I}$ & 5877.29 \\
\hline $6085.90 \pm 2.00 \ldots \ldots$ & 6064 & 6107 & $0.113 \pm 0.016$ & $0.71 \pm 0.10$ & 3.78 & -0.95 & {$[\mathrm{Fe}$ VII $]$} & 6087.98 \\
\hline $6303.05 \pm 0.53 \ldots \ldots$ & 6283 & 6326 & $0.179 \pm 0.016$ & $1.15 \pm 0.11$ & 3.14 & -0.64 & {$\left[\begin{array}{ll}\mathrm{O} & \mathrm{I}\end{array}\right]$} & 6302.05 \\
\hline $6370.46 \pm 2.67 \ldots \ldots$ & 6347 & 6400 & $0.217 \pm 0.018$ & $1.36 \pm 0.11$ & 10.18 & -0.09 & {$\left[\begin{array}{ll}\mathrm{O} & \mathrm{I}\end{array}\right]$} & 6365.54 \\
\hline & $\ldots$ & $\ldots$ & $\ldots$ & $\ldots$ & $\ldots$ & $\ldots$ & {$[\mathrm{Fe} \mathrm{x}]$} & 6376.30 \\
\hline $6551.06 \pm 1.24 \ldots \ldots$ & 6544 & 6556 & $0.195 \pm 0.029$ & $0.43 \pm 0.06$ & 2.21 & -0.20 & {$[\mathrm{~N}$ II $]$} & 6549.85 \\
\hline $6564.93 \pm 0.22 \ldots \ldots$ & 6400 & 6765 & $30.832 \pm 0.098$ & $194.52 \pm 0.62$ & 47.39 & 0.35 & $\mathrm{H} \alpha$ & 6564.61 \\
\hline $6585.64 \pm 0.34 \ldots \ldots$ & 6577 & 6593 & $0.831 \pm 0.034$ & $2.02 \pm 0.08$ & 2.65 & -0.02 & {$[\mathrm{~N}$ II $]$} & 6585.28 \\
\hline $6718.85 \pm 0.46 \ldots \ldots$ & 6708 & 6726 & $0.276 \pm 0.014$ & $1.65 \pm 0.08$ & 3.09 & -0.27 & {$[\mathrm{~S}$ II $]$} & 6718.29 \\
\hline $6733.72 \pm 0.46 \ldots \ldots$ & 6726 & 6742 & $0.244 \pm 0.013$ & $1.49 \pm 0.08$ & 2.54 & -0.11 & {$[\mathrm{~S}$ II $]$} & 6732.67 \\
\hline $7065.67 \pm 2.92 \ldots \ldots$ & 7034 & 7105 & $0.451 \pm 0.026$ & $3.06 \pm 0.18$ & 15.23 & 0.05 & $\mathrm{He} \mathrm{I}$ & 7067.20 \\
\hline $7138.73 \pm 1.12 \ldots \ldots$ & 7131 & 7148 & $0.082 \pm 0.013$ & $0.57 \pm 0.09$ & 3.13 & -0.01 & {$[\mathrm{Ar} \mathrm{III}]$} & 7137.80 \\
\hline $7321.27 \pm 3.55 \ldots \ldots$ & 7285 & 7360 & $0.359 \pm 0.031$ & $2.52 \pm 0.22$ & 14.26 & 0.30 & {$[\mathrm{O} \mathrm{II}]$} & 7321.48 \\
\hline $7890.49 \pm 3.33 \ldots \ldots$ & 7883 & 7897 & $0.096 \pm 0.018$ & $0.69 \pm 0.13$ & 2.97 & 0.39 & {$[\mathrm{Ni} \mathrm{III}]$} & 7892.10 \\
\hline & $\ldots$ & $\ldots$ & $\ldots$ & $\ldots$ & $\ldots$ & $\ldots$ & {$[\mathrm{Fe} \mathrm{XI}]$} & 7894.00 \\
\hline
\end{tabular}

a Ions ending with a colon (:) are uncertain identifications.

b Multiplet designations for Fe are from Netzer \& Wills 1983, Grandi 1981, and Phillips 1978.

c Broad feature composed mainly of Fe II multiplets. 


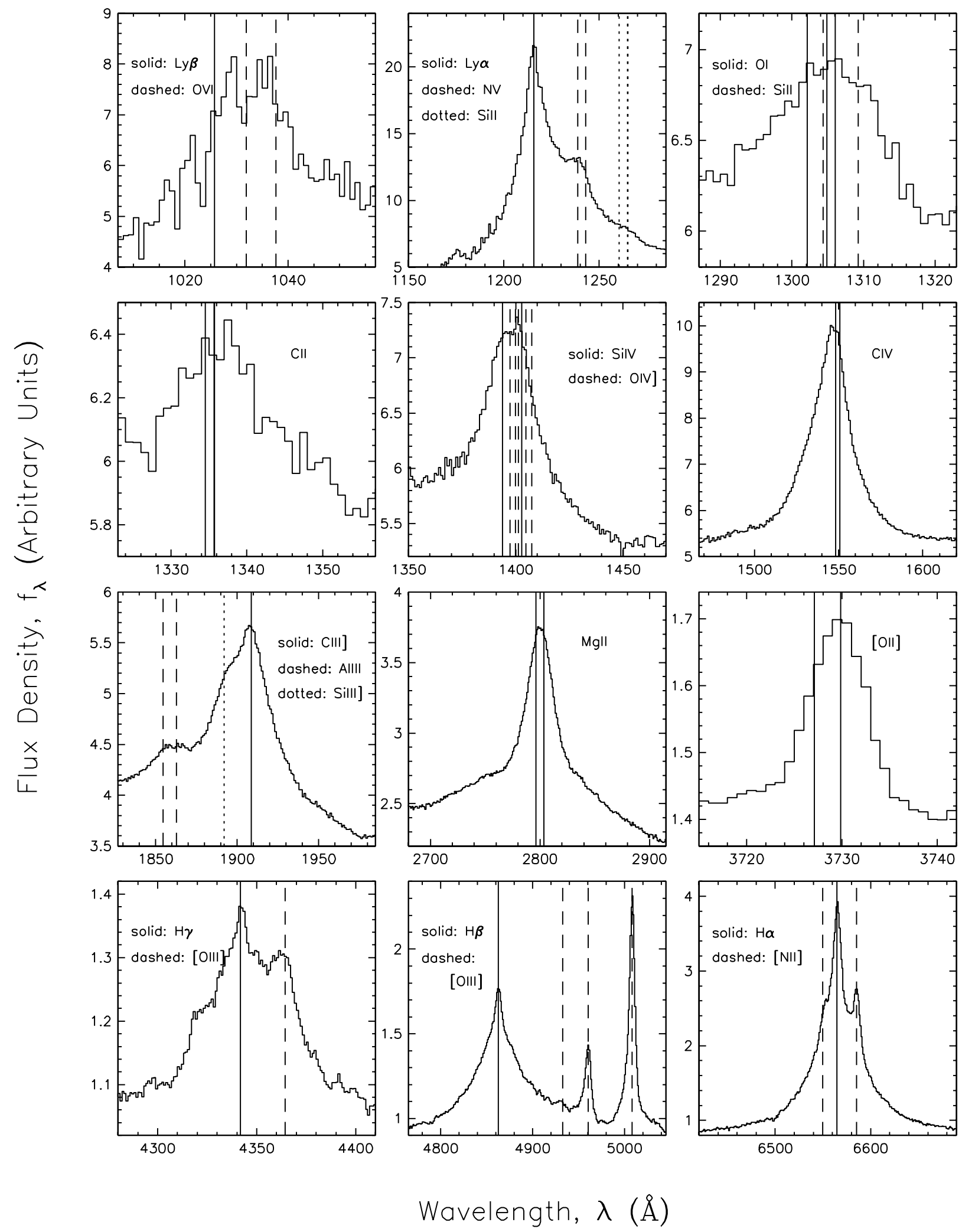

FIG. 7.-Detailed view of 12 strong emission-line regions. The laboratory rest-wavelength positions of the major line components are shown. Many of the emission lines are composed of blended multiple transitions (doublets, triplets, etc.) from the same ion.

apparent "shifts" that can arise from several sources, including contamination by line blends, incorrect identifications, and line asymmetry. To minimize these problems, we have selected only relatively strong lines with isolated peaks, and we have remeasured the peaks of only the top
$25 \%$ of the line flux for lines that appeared to have a very broad component or asymmetric profile. The velocity shifts for the selected lines are listed in Table 4. A negative velocity indicates that a line is blueshifted with respect to the nominal laboratory wavelength and vice versa. By design, 
TABLE 3

Composite Quasar Absorption-Line Features

\begin{tabular}{|c|c|c|c|c|}
\hline $\begin{array}{l}\lambda_{\text {obs }} \\
(\AA)\end{array}$ & $\begin{array}{l}W \\
(\AA)\end{array}$ & $\begin{array}{l}\text { Width } \sigma_{\lambda} \\
(\AA)\end{array}$ & ID & $\begin{array}{l}\lambda_{\text {lab }} \\
(\AA)\end{array}$ \\
\hline \multicolumn{5}{|c|}{ Median Composite Using All Spectra } \\
\hline $3800.38 \pm 1.09 \ldots \ldots$ & $0.35 \pm 0.03$ & 4.14 & H10 & 3798.98 \\
\hline $3837.12 \pm 1.49 \ldots \ldots$ & $0.46 \pm 0.03$ & 5.96 & H9 & 3836.47 \\
\hline $3934.96 \pm 0.55 \ldots \ldots$ & $0.91 \pm 0.03$ & 7.11 & $\mathrm{Ca}$ II & 3934.78 \\
\hline $8502.80 \pm 7.22 \ldots \ldots$ & $1.11 \pm 0.61$ & 3.85 & $\mathrm{Ca}$ II & 8500.36 \\
\hline $8544.17 \pm 1.89 \ldots \ldots$ & $2.22 \pm 0.44$ & 3.87 & $\mathrm{Ca}$ II & 8544.44 \\
\hline \multicolumn{5}{|c|}{ Low-Redshift Median Composite $\left(z_{\mathrm{em}} \leq 0.5\right)$} \\
\hline $3737.82 \pm 1.03 \ldots \ldots$ & $0.16 \pm 0.03$ & 0.97 & H13: & 3735.43 \\
\hline $3749.45 \pm 1.13 \ldots \ldots$ & $0.31 \pm 0.04$ & 2.96 & H12: & 3751.22 \\
\hline $3774.09 \pm 1.27 \ldots \ldots$ & $0.36 \pm 0.04$ & 3.56 & H11 & 3771.70 \\
\hline $3799.71 \pm 0.89 \ldots \ldots$ & $0.84 \pm 0.05$ & 4.86 & H10 & 3798.98 \\
\hline $3837.77 \pm 1.16 \ldots \ldots$ & $0.95 \pm 0.05$ & 5.69 & H9 & 3836.47 \\
\hline $3934.94 \pm 0.48 \ldots \ldots$ & $1.64 \pm 0.06$ & 6.94 & $\mathrm{Ca}$ II & 3934.78 \\
\hline $3974.66 \pm 0.88 \ldots \ldots$ & $0.36 \pm 0.04$ & 2.42 & $\mathrm{Ca} \mathrm{II}$ & 3969.59 \\
\hline \multirow[t]{2}{*}{$5892.66 \pm 1.24 \ldots \ldots$} & $0.44 \pm 0.05$ & 3.72 & $\mathrm{Na}$ II & 5891.58 \\
\hline & $\ldots$ & $\cdots$ & $\cdots$ & 5897.56 \\
\hline $8502.80 \pm 7.22 \ldots \ldots$ & $1.11 \pm 0.61$ & 3.85 & $\mathrm{Ca}$ II & 8500.36 \\
\hline $8544.17 \pm 1.89 \ldots \ldots$ & $2.22 \pm 0.44$ & 3.87 & $\mathrm{Ca}$ II & 8544.44 \\
\hline
\end{tabular}

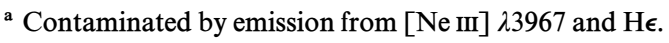
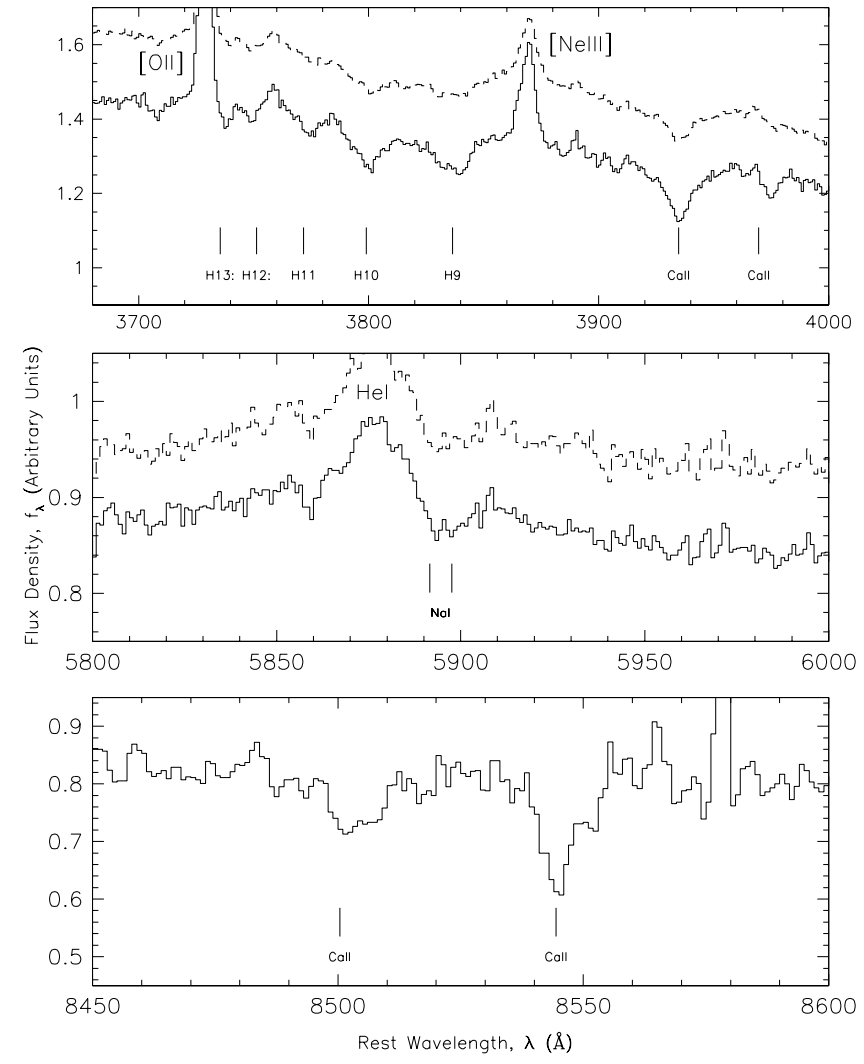

FIG. 8.-Detailed view of absorption-line regions in the low-redshift composite quasar spectrum (solid line). The laboratory rest-wavelength positions of detected absorption lines are labeled by ion. Several strong emission lines are also labeled. The Ca II $\lambda 3968$ line is contaminated by emission from [Ne III] $\lambda 3967$ and $\mathrm{H} \epsilon$. The full sample composite spectrum (dashed line, offset) is also shown for comparison in the top two panels (the spectra are identical in the wavelength region covered in the bottom panel). the $[\mathrm{O}$ III] $\lambda 5007$ line peak shows no shift from its laboratory rest wavelength to well within the measurement uncertainty. All other emission-line peaks are measured with respect to the rest frame of the [O III] $\lambda 5007$ line. The two other measurable [O $\mathrm{III}]$ lines, $\lambda 4363$ and $\lambda 4958$, have no velocity shift to within the uncertainties.

It has been suggested that there is a correlation between the line shift and the ionization energy of the species (see, e.g., Tytler \& Fan 1992; McIntosh et al. 1999). Quasar emission lines are generally separated into two broad categories: the permitted and semiforbidden lines, which are typically broad (FWHM $>500 \mathrm{~km} \mathrm{~s}^{-1}$ ), and the much narrower forbidden lines. These classes of lines are thought to arise from physically distinct regions: the parsec-scale broad-line region (BLR) and the kiloparsec-scale narrow-line region (NLR), respectively. Since their origins are likely to be different, we treat the BLR and NLR lines separately. Figure 9 shows the ionization energy versus velocity shifts in Table 4 for both the BLR and the NLR lines, labeled by their ions. In both cases, there is an apparent anticorrelation between the velocity shifts and ionization potential in Figure 9. The Spearman rank correlation coefficient for the BLR lines gives a random probability of finding as strong a correlation at about $0.6 \%$. We have taken the uncertainties in the velocity measurements into account by creating $10^{4}$ mock data sets of velocities, randomly distributed for each emission line according to the velocity uncertainties, and then recalculated the correlation probabilities. For half of the mock data sets, the random probability of a correlation was less than $1.6 \%$ for the BLR lines. Thus, we would have found a significant anticorrelation between the velocity offsets and ionization potentials for a majority of independent measurements. The low-ionization C II $\lambda 1335$ line has the maximum redshift at $292 \mathrm{~km} \mathrm{~s}^{-1}$, and the high- 
TABLE 4

Emission-Line Velocity Shifts Relative to [O III] $\lambda 5007$

\begin{tabular}{|c|c|c|c|c|c|}
\hline Ion & $\begin{array}{l}\lambda_{\text {lab }} \\
(\AA)\end{array}$ & $\begin{array}{l}\lambda_{\text {obs }} \\
(\AA)\end{array}$ & $\begin{array}{l}\Delta \lambda \\
(\AA)\end{array}$ & $\begin{array}{l}\text { Velocity } \\
\left(\mathrm{km} \mathrm{s}^{-1}\right)\end{array}$ & $\begin{array}{l}\text { Ionization Energy } \\
(\mathrm{eV})\end{array}$ \\
\hline $\operatorname{Ly} \alpha \ldots \ldots$ & 1215.67 & 1216.25 & 0.58 & $143 \pm 91$ & 13.60 \\
\hline $\mathrm{N} v \ldots . \ldots .$. & 1240.14 & 1239.85 & -0.29 & $-70 \pm 162$ & 97.89 \\
\hline $\mathrm{C}$ IV $\ldots \ldots \ldots$ & 1549.06 & 1546.15 & -2.91 & $-563 \pm 27$ & 64.49 \\
\hline He II ........ & 1640.42 & 1637.84 & -2.58 & $-471 \pm 151$ & 54.42 \\
\hline $\mathrm{N}$ III $] \ldots \ldots$. & 1750.26 & 1748.31 & -1.95 & $-334 \pm 128$ & 47.45 \\
\hline Al III .......... & 1857.40 & 1856.76 & -0.64 & $-103 \pm 190$ & 18.83 \\
\hline $\mathrm{C}$ III $] \ldots \ldots \ldots$ & 1908.73 & 1907.30 & -1.43 & $-224 \pm 28$ & 47.89 \\
\hline $\operatorname{Mg}$ II $\ldots \ldots \ldots$ & 2798.75 & 2800.26 & 1.51 & $161 \pm 10$ & 15.04 \\
\hline$[\mathrm{Ne} \mathrm{v}] \ldots \ldots$ & 3346.82 & 3345.39 & -1.43 & $-128 \pm 67$ & 126.22 \\
\hline$[\mathrm{Ne} v] \ldots \ldots$ & 3426.84 & 3426.17 & -0.67 & $-58 \pm 38$ & 126.22 \\
\hline$[\mathrm{O} \mathrm{II}] \ldots \ldots \ldots$ & 3728.48 & 3729.66 & 1.18 & $94 \pm 14$ & 35.12 \\
\hline$[\mathrm{Fe}$ VII $] \ldots \ldots$ & 3759.99 & 3758.46 & -1.53 & $-122 \pm 44$ & 125.00 \\
\hline$[\mathrm{Ne}$ III $] \ldots . .$. & 3869.85 & 3869.77 & -0.08 & $-6 \pm 19$ & 63.46 \\
\hline $\mathrm{H} \delta \ldots \ldots \ldots$ & 4102.89 & 4102.73 & -0.16 & $-11 \pm 48$ & 13.60 \\
\hline $\mathrm{H} \gamma \ldots \ldots \ldots$ & 4341.68 & 4342.02 & 0.34 & $23 \pm 30$ & 13.60 \\
\hline$[\mathrm{O}$ III $] \ldots \ldots$ & 4364.44 & 4364.15 & -0.29 & $-19 \pm 48$ & 54.94 \\
\hline $\mathrm{H} \beta \ldots \ldots \ldots$ & 4862.68 & 4862.66 & -0.02 & $-1 \pm 14$ & 13.60 \\
\hline$[\mathrm{O}$ III $] \ldots \ldots$ & 4960.30 & 4960.36 & 0.06 & $3 \pm 13$ & 54.94 \\
\hline$[\mathrm{O}$ III $] \ldots \ldots$ & 5008.24 & 5008.22 & -0.02 & $-1 \pm 10$ & 54.94 \\
\hline$[\mathrm{Fe}$ VII $] \ldots \ldots$ & 5160.33 & 5160.81 & 0.48 & $27 \pm 54$ & 125.00 \\
\hline$[\mathrm{Fe}$ VII $] \ldots \ldots$ & 5722.30 & 5722.27 & -0.03 & $-1 \pm 110$ & 125.00 \\
\hline He I ......... & 5877.29 & 5876.75 & -0.54 & $-27 \pm 56$ & 24.59 \\
\hline$[\mathrm{Fe}$ VII $] \ldots \ldots$ & 6087.98 & 6086.90 & -1.08 & $-53 \pm 151$ & 125.00 \\
\hline$[\mathrm{O}$ I $] \ldots \ldots \ldots$ & 6302.05 & 6303.05 & 1.00 & $47 \pm 25$ & 13.62 \\
\hline$[\mathrm{N} \mathrm{III}] \ldots \ldots \ldots$ & 6549.85 & 6551.06 & 1.21 & $55 \pm 56$ & 29.60 \\
\hline $\mathrm{H} \alpha \ldots \ldots \ldots$ & 6564.61 & 6565.22 & 0.61 & $27 \pm 13$ & 13.60 \\
\hline$[\mathrm{N} \mathrm{III}] \ldots \ldots \ldots$ & 6585.28 & 6585.64 & 0.36 & $16 \pm 15$ & 29.60 \\
\hline$[\mathrm{S}$ II $] \ldots \ldots \ldots$ & 6718.29 & 6718.85 & 0.56 & $25 \pm 20$ & 23.33 \\
\hline$[\mathrm{S}$ III $] \ldots \ldots \ldots$ & 6732.67 & 6733.72 & 1.05 & $46 \pm 20$ & 23.33 \\
\hline$[$ Ar III $] \ldots . .$. & 7137.80 & 7138.73 & 0.93 & $39 \pm 47$ & 40.74 \\
\hline
\end{tabular}

ionization $\mathrm{C}$ IV $\lambda 1549$ line has the maximum blueshift at $-564 \mathrm{~km} \mathrm{~s}^{-1}$. The $\mathrm{N} v$ point appears to be somewhat of an outlier, possibly because of severe blending with Ly $\alpha$. It is also interesting that $\mathrm{N} v$ does not follow the Baldwin effect (Espey \& Andreadis 1999), the strength of which is otherwise anticorrelated with ionization potential. In any case, the rank correlation of the velocity offset and ionization potential is not significantly stronger when $\mathrm{N} v$ is removed, and we have no compelling reason to do so. The velocity offsets are not as strong for the NLR lines- $\lesssim 100 \mathrm{~km} \mathrm{~s}^{-1}$ -but the Spearman rank correlation probability is $1.3 \times 10^{-4}$, which is quite significant, and we find the probability is less than $1 \%$ for half of the mock data sets. We discuss emission-line velocity shifts further in $\S 5$.

\subsection{Spectrum-to-Spectrum Differences}

While constructing the median composite, the flux levels of overlapping spectra were scaled so that the integrated flux densities were the same. Thus, we expect the variation in the continuum flux density across the spectrum to reflect the spectrum-to-spectrum differences caused by differing continuum shapes and emission-line fluxes and profiles. (This does not, however, address spectral time variability.) Figure 10 shows the $68 \%$ semi-interquantile range divided by the median spectrum, after the contribution from the combined flux density uncertainties of each spectrum were removed in quadrature. The individual spectral uncertainties include statistical noise estimates but not uncertainties in the (unfinalized) flux calibration. The largest relative variations from the median spectrum occur in the narrow emission lines, such as the [O III] $\lambda \lambda 4958,5007$ lines, and the cores of broad emission lines, such as C IV $\lambda 1549$ and $\mathrm{Ly} \alpha \lambda 1215$. Variations of the broad components of $\mathrm{H} \alpha$ $\lambda 6563$ and $\mathrm{Mg}$ II $\lambda 2798$ are evident but less so for $\mathrm{H} \beta \lambda 4861$ and $\mathrm{C}$ IV $\lambda 1549$, and there is little sign of variation in the C III $\lambda 1908$ line. Most of the broad Fe II complexes show significant variation. The Ly $\alpha$ forest region varies considerably, as expected, since structure in the forest can be partly resolved in the individual spectra, and the forest strength changes with redshift. As a result, the combination of spectra at different redshifts will naturally give rise to a high variance. An additional feature of some interest is the pair of variation peaks at 3935 and $3970 \AA$, which correspond precisely to the $\mathrm{Ca}$ II doublet. These are detected in absorption in the median composites (although [Ne III] and $\mathrm{H} \epsilon$ emission interfere with Ca II 23970 ), and the variation may indicate that spectral contamination by the host galaxy is fairly common. A full analysis of spectrum-to-spectrum variations requires other means, such as principal component analysis (Boroson \& Green 1992; Francis et al. 1992; Brotherton et al. 1994a; Wills et al. 1999), which we plan for a future project.

\section{DISCUSSION}

The Large Bright Quasar Survey (LBQS) composite (kindly provided by S. Morris) updated from Francis et al. (1991), the First Bright Quasar Survey (FBQS) composite (available electronically; Brotherton et al. 2001), and our median composite are shown for comparison in Figure 11. The spectra have been scaled to unit-average flux density in 


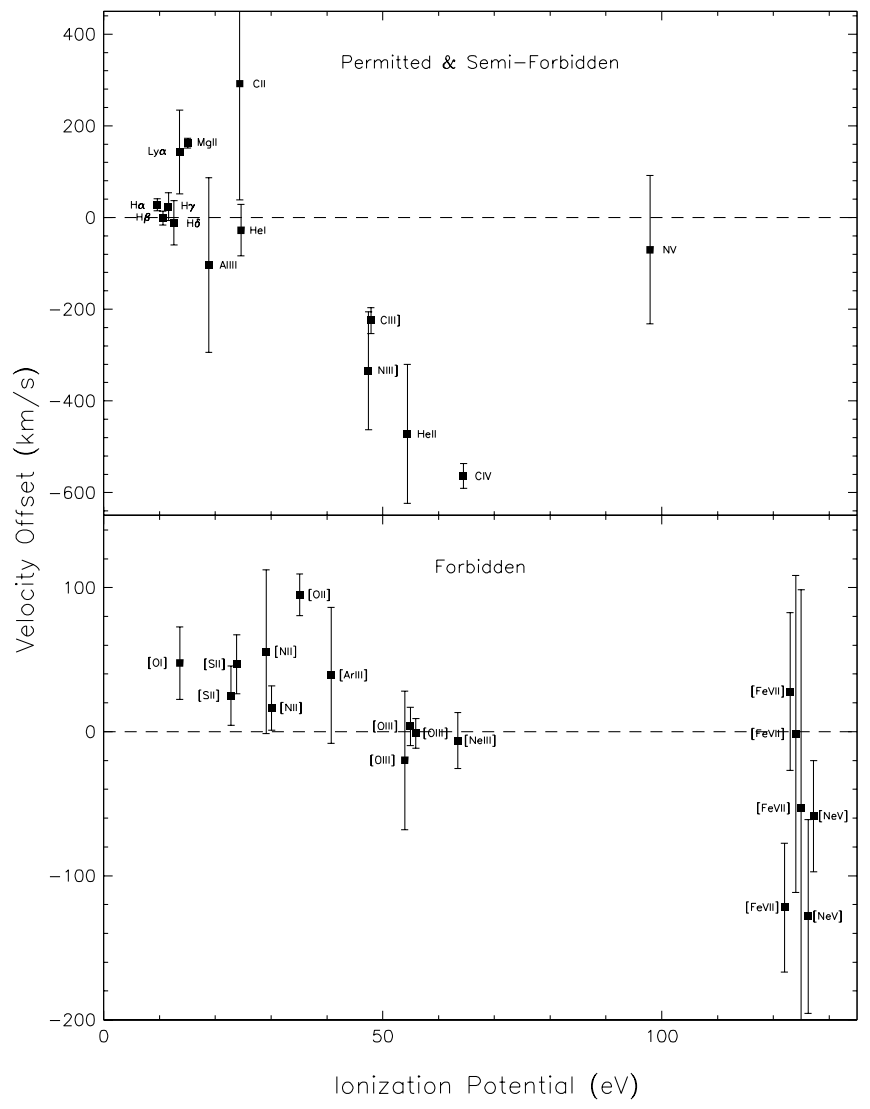

FIG. 9.-Emission-line velocity offsets relative to laboratory rest wavelengths as a function of ionization potential for selected emission lines. Error bars show the $1 \sigma$ uncertainty in the velocity measurement. The points are labeled by ion. Ionization potentials corresponding to the same ion are slightly offset from each other for clarity. Permitted and semiforbidden lines are shown in the top panel, and forbidden lines are shown in the bottom panel.

the range $3020-3100 \AA$. All three spectra are quite similar in appearance except for slight differences. The strength of the Ly $\alpha$ line and some of the narrow emission lines in the FBQS composite are stronger than for the other composites. The difference is probably due to the fact that the FBQS sample is entirely radio selected, and there is a correlation between

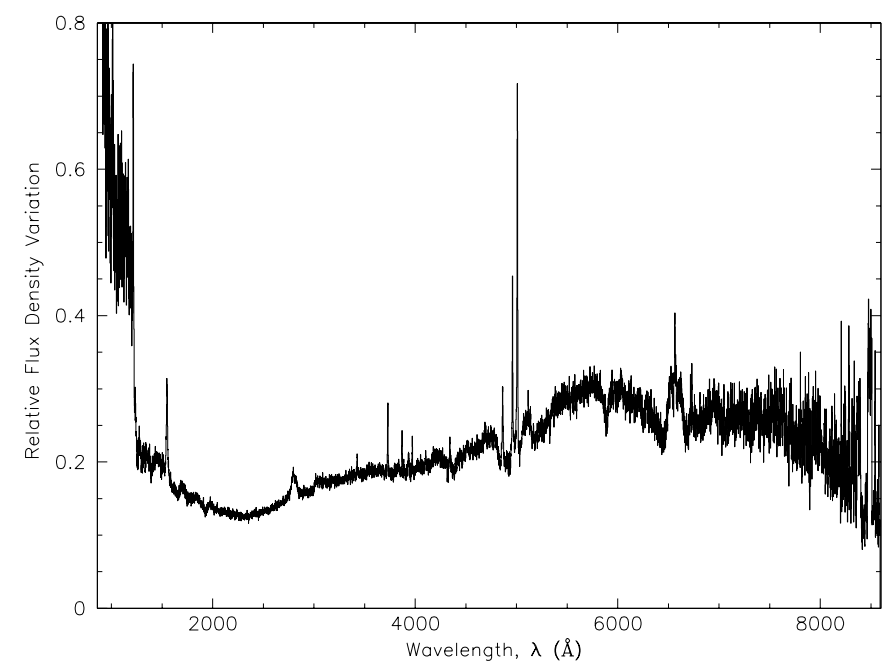

FIG. 10.-Spectrum-to-spectrum variation of the quasar composite flux density relative to the median flux as a function of rest wavelength.

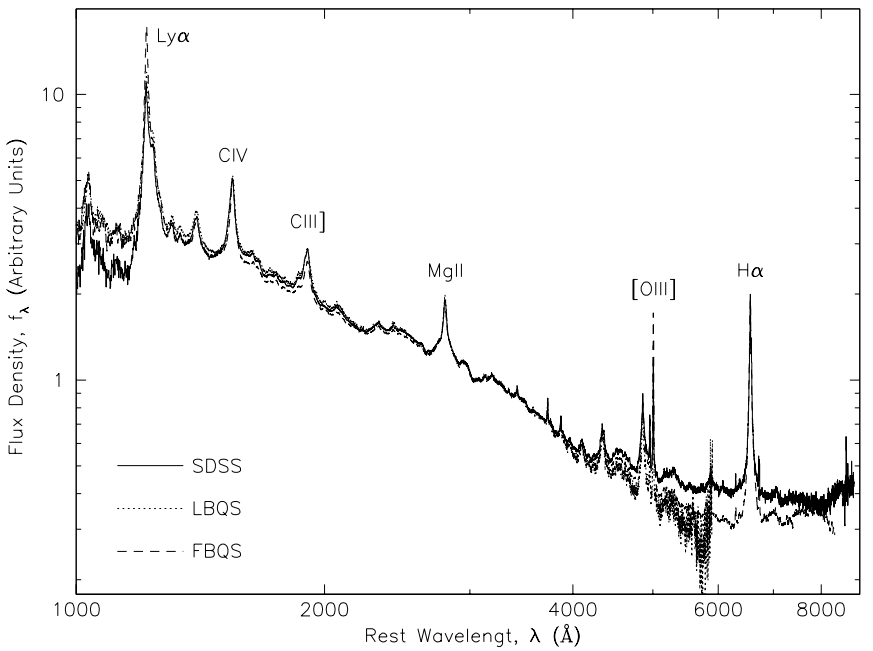

FIG. 11.-Comparison of the SDSS median quasar composite spectrum (solid line) with the LBQS (dotted line) and FBQS (dashed line) composites. The spectra are scaled to the same average flux density between 3020 and 3100 A. Several major emission lines are labeled for reference.

line strengths and radio loudness (Boroson \& Green 1992; Francis et al. 1992; Brotherton et al. 1994a; Wills et al. 1999). Otherwise, the relative fluxes are similar for the lines in common among the various composites. The higher resolution and higher $\mathrm{S} / \mathrm{N}$ of our composite has allowed us to identify many more lines than listed for the other spectra (although a number of the features we find are present at a lower significance level in the other spectra). We have identified a total of 85 emission features in the median spectrum. All the features have been identified in other quasar or AGN spectra but not in any single object. A large number of the identified features are attributed to either $\mathrm{Fe}$ II or $\mathrm{Fe}$ III multiplets. The combination of these features has been shown to greatly affect the color-redshift relationship for quasars (Richards et al. 2001b).

A single power law is an adequate fit to the continuum between $\mathrm{Ly} \alpha$ and $\mathrm{H} \beta$, especially given the predicted strengths of the $\mathrm{Fe}$ II and $\mathrm{Fe}$ III emission-line complexes in that range (Verner et al. 1999; Netzer \& Wills 1983; Laor et al. 1997). The index we find, $\alpha_{v}=-0.44$, is in good agreement with most recent values found in optically selected quasar samples. Table 5 lists average power-law indices from various sources over the past decade. The LBQS, FBQS, and Hubble Space Telescope (HST) composite (Zheng et al. 1997) spectra are available electronically, so for consistency, we have also remeasured the power-law indices of those spectra using the technique described in $\S$ 4.1. The remeasured values are not significantly different from the values given in previous papers.

Most of the composite measurements agree with averages over continuum fits to individual spectra. One outlier is the measurement by Zheng et al. (1997), who find a steeper (redder) continuum with $\alpha_{v}=-0.99$ using a composite made with spectra from HST. The difference is attributed to the lower redshift of the Zheng et al. (1997) quasar sample and a correlation between redshift and steeper UV continuum (Francis 1993). To test this, we have created a lowredshift geometric mean composite using only those quasars that cover a rest wavelength of $5000 \AA(z<0.84)$. Since the $1350 \AA$ 皮 wavelength region we have used to measure continuum slopes is not covered by the low- $z$ com- 
TABLE 5

Measurements of the Optical Power-Law CONTINUUm INDEX For Quasars

\begin{tabular}{|c|c|c|c|c|c|}
\hline$\alpha_{v}$ & Sample Selection & Measurement Method & Redshift Range & Median Redshift & Source \\
\hline$-0.44 \ldots \ldots$ & Optical and radio & Composite spectrum & $0.04-4.79$ & 1.25 & 1 \\
\hline$-0.93 \ldots \ldots$ & Optical & Average value from spectra & $3.58-4.49$ & 3.74 & 2 \\
\hline$-0.46 \ldots \ldots$ & Radio & Composite spectrum & $0.02-3.42$ & 0.80 & 3 \\
\hline$-0.43 \ldots \ldots$ & Radio & Composite spectrum (remeasure) & $0.02-3.42$ & 0.80 & 1,3 \\
\hline$-0.39 \ldots \ldots$ & Radio & Photometric estimates & $0.38-2.75$ & 1.22 & 4 \\
\hline$-0.33 \ldots \ldots$ & Optical & Average value from spectra & $0.12-2.17$ & 1.11 & 5 \\
\hline$-0.99 \ldots \ldots$ & Optical and radio & Composite spectrum & $0.33-3.67$ & 0.93 & 6 \\
\hline$-1.03 \ldots \ldots$ & Optical and radio & Composite spectrum (remeasure) & $0.33-3.67$ & 0.93 & 1,6 \\
\hline$-0.46 \ldots \ldots$ & Optical & Photometric estimates & $0.44-3.36$ & 2.00 & 7 \\
\hline$-0.32 \ldots \ldots$ & Optical & Composite spectrum & $\mathrm{NA}^{\mathrm{a}}$ & 1.3 & 8 \\
\hline$-0.36 \ldots \ldots$ & Optical & Composite spectrum (remeasure) & $\mathrm{NA}^{\mathrm{a}}$ & 1.3 & 1,8 \\
\hline$-0.67 \ldots \ldots$ & Optical & Composite spectrum & $0.16-3.78$ & 1.51 & 9 \\
\hline$-0.70 \ldots \ldots$ & Radio & Composite spectrum & $\mathrm{NA}^{\mathrm{a}}$ & $\mathrm{NA}^{\mathrm{a}}$ & 9 \\
\hline
\end{tabular}

a The value was not given in the reference or derivable from the data.

REFERENCES. - (1) This paper; (2) Schneider et al. 2001; (3) Brotherton et al. 2001; (4) Carballo et al. 1999; (5) Natali et al. 1998; (6) Zheng et al. 1997; (7) Francis 1996; (8) Francis et al. 1991; (9) Cristiani \& Vio 1990.

posite, we used instead the flux density in the wavelength range $3020-3100 \AA$ multiplied by a factor of 0.86 , which is the ratio of the flux density of the power-law fit to the flux density of the spectrum for the full sample geometric mean composite in that range. We find a steeper index for the low-redshift composite, $\alpha_{v}=-0.65$, than for the full-sample composite, $\alpha_{v}=-0.44$, although the difference is not as great as with the Zheng et al. (1997) composite.

Another apparently discrepant value is the result from Schneider et al. (2001), who find $\alpha_{v}=-0.93$ for a sample of very high-redshift quasars. Similar values for high- $z$ samples have been found by Fan et al. (2001) and Schneider, Schmidt, \& Gunn (1991). The steep indices measured for high- $z$ quasars may be due to the restricted wavelength range typically used in fitting the continua, as suggested by Schneider et al. (2001), and not to a change in the underlying spectral index at high redshift. At high redshifts, only relatively short wavelength ranges redward of $L y \alpha$ are available in optical spectra, and these tend to be populated by broad $\mathrm{Fe}$ II and $\mathrm{Fe}$ III complexes. If, for example, the regions of the median composite near 1350 and $1600 \AA$ (just redward of the $\mathrm{C}$ IV emission line) are taken as continuum (as Schneider et al. 2001 did), we find a power-law index of $\alpha_{v}=-0.93$. This example demonstrates the generic difficulty of measuring continuum indices without a very large range of wavelength, or some estimate of the strength of the contribution from blended emission lines.

The continuum slope changes abruptly near $5000 \AA$ and becomes steeper with an index of $\alpha_{v}=-2.45$, which is a good fit up to the red end of the spectrum ( $8555 \AA$ ). This change is also evident in the FBQS composite and has been noted in the spectra of individual quasars (Wills et al. 1985). An upturn in the spectral energy distribution of quasarsthe so-called near-infrared inflection, presumably caused by emission from hot dust - has been seen starting between 0.7 and $1.5 \mu \mathrm{m}$ (see, e.g., Neugebauer et al. 1987; Elvis et al. 1994). This may be, in part, what we are seeing at wavelengths beyond $\approx 5000 \AA$, but it is unlikely that the sublimation temperature of dust would be high enough for the emission to extend to wavelengths below $6000 \AA$ (Puget, Leger, \& Boulanger 1985; Efstathiou, Hough, \& Young 1995).

Another possible contributor to the long-wavelength steepening is contamination from the host galaxies. The $3^{\prime \prime}$ optical fiber diameter subtends much if not all of the hostgalaxy image, even for the lowest redshift quasars. The best evidence for the contribution of host-galaxy light is the presence of stellar absorption lines in the composite spectra. The lines become stronger as the redshift and, equivalently, the luminosity distributions of the quasar sample are lowered. This is seen by comparing the absorption-line strengths of the low-redshift median composite $(\S 3)$ with the full-sample composite. The strengths of the absorption lines in the low-redshift median composite, assuming a typical elliptical galaxy spectrum, imply a contribution to the composite quasar light from stars of about $7 \%-15 \%$ at the locations of $\mathrm{Ca}$ II $\lambda 3933$ and $\mathrm{Na}$ I $\lambda 5896$ and about $30 \%$ at the locations of $\mathrm{Ca}$ II $\lambda \lambda 8498,8542$. The trend of a greater contribution from starlight with increasing wavelength is expected because the least luminous quasars, in which the relative host-galaxy light is presumably most important, contribute the majority of spectra to the composite at longer wavelengths. This trend has also been seen in the spectral light from the nuclei of individual low-redshift Seyfert galaxies and other AGNs (Terlevich, Diaz, \& Terlevich 1990; Serote Roos et al. 1998), which suggests a significant contribution from starburst activity dominated by red supergiants (Cid Fernandes \& Terlevich 1995). The mean absolute $r^{\prime}$ magnitude of the quasars making up the low- $z$ composite is $M_{r^{\prime}}=-21.7$ (Fig. 1), which implies a hostgalaxy magnitude of about $M_{r^{\prime}}=-19.2$ (assuming a host contribution of $\sim 10 \%$ ) - a moderately luminous value in the SDSS filter system (Blanton et al. 2001). We conclude that both stellar light from the host galaxies and a real change in the quasar continuum cause the steepening of the spectral index beyond $5000 \AA$.

The detection of stellar Balmer absorption lines implies that young or intermediate-age stars make a substantial contribution to the light of the host galaxies. This is at odds with the conclusion, based on host-galaxy spectra (Nolan et al. 2001) and two-dimensional image modeling (McLure et al. 1999; McLure \& Dunlop 2000), that the hosts of quasars and radio galaxies are "normal" giant elliptical galaxies. The discrepancy cannot immediately be attributed to redshift differences, since the McLure \& Dunlop (2000) sample extends to $z \approx 1$, and we detect Balmer absorption lines in the full-sample composite with a mean redshift of $z=1.25$. More likely, the difference is due to the fact that our spectra 
include only the inner $3^{\prime \prime}$ of the galaxy light, while the spectra taken by Nolan et al. (2001) sample only off-nuclear ( $5^{\prime \prime}$ from nucleus) light, and the image modeling includes the entire profile of the galaxies. This suggests that the stellar population near the nuclei of quasar host galaxies - near the quasars themselves - is substantially younger than that of the host galaxies.

Velocity shifts in the BLR lines relative to the forbidden NLR lines - taken to be at the systemic host-galaxy redshift - are seen for most quasars and are similar to the values we find for the composite BLR lines relative to [O III] $\lambda 5007$ (see, e.g., Tytler \& Fan 1992; Laor et al. 1995; McIntosh et al. 1999). The origin of the shifts is not known, but explanations include gas inflows and outflows (see, e.g., Gaskell 1982; Corbin 1990), attenuation by dust (Grandi 1977; Heckman et al. 1981), relativistic effects (Netzer 1977; Corbin 1995, 1997; McIntosh et al. 1999), and line emission from physically different locations (see, e.g., Espey et al. 1989). The magnitudes of the shifts seem to depend upon the ionization energies (Gaskell 1982; Wilkes 1986; Espey et al. 1989; Tytler \& Fan 1992; McIntosh et al. 1999), in the sense that more negative velocities (blueshifts) are seen for higher ionization lines. We have confirmed this correlation using a large number of BLR lines (\$ 4.3).

It is often assumed that the NLR lines are at the systemic redshift of the quasar, since the lines are thought to originate in a kiloparsec-scale region centered on the quasar, and the lines show good agreement (to within $100 \mathrm{~km} \mathrm{~s}^{-1}$ ) with the redshifts of host galaxies determined by stellar absorption lines (Gaskell 1982; Vrtilek \& Carleton 1985) and $\mathrm{H}_{\text {I }} 21 \mathrm{~cm}$ observations (Hutchings, Gower, \& Price 1987). However, for some of the higher ionization forbidden lines, such as [O III] $\lambda 5007, \mathrm{Ne} v \quad \lambda 3426, \mathrm{Fe}$ VII $\lambda 6086, \mathrm{Fe} \mathrm{x}$ $\lambda 6374$, and $\mathrm{Fe} \mathrm{XI} \lambda 7892$, seen in quasars and Seyfert galaxies, significant velocity shifts, usually blueshifts, have been detected in the past (e.g., Heckman et al. 1981; Mirabel \& Wilson 1984; Penston et al. 1984; Whittle 1985; Appenzeller \& Wagner 1991). The large number (17) of NLR lines we have been able to measure cover a wide range in ionization potentials. These lines are shifted with respect to one another, and the shifts are correlated with ionization energy. This appears to be a real effect, since we have been careful to select only those lines that have well-defined nonblended peaks. Another verification of the accuracy of the velocity measurements is that lines originating from the same ion but at different rest wavelengths almost always have consistent velocity offsets within the measurement uncertainties (Table 4 and Fig. 9).

The NLR velocity shifts and their correlation with ionization potential suggest that the same mechanism responsible for the shifts of the BLR lines also applies to the NLR lines, although the effect is weaker. One possible explanation is that the BLR contains some lower density, forbidden-lineemitting gas, as first suggested by Penston (1977). The correlation is strong, but the effect is subtle, so follow-up work will likely have to involve both higher quality optical spectra and observations in the near-IR in order to detect a sufficient sample of narrow forbidden lines.

We have implicitly assumed that the velocity differences are independent of other factors, such as redshift and luminosity. However, McIntosh et al. (1999) found that higher $z$ quasars tend to have greater velocity offsets relative to the [O III] line. For quasars in our sample with $z>0.84$, the [O III] emission line is redshifted out of the spectra, which is why we used a cross-correlation technique to estimate the center-of-mass redshifts. If the true velocity offsets depend upon redshift, the relation will be weakened by the crosscorrelation matching, which finds the best match to a lower redshift template, and thus will tend to yield the lower redshift emission-line positions. A desirable future project is extending the wavelength coverage to the near-infrared at higher redshift and to the ultraviolet at lower redshift in order to simultaneously detect low- and high-velocity lines. Such a program of even a relatively modest sample size would be highly beneficial to many quasar studies.

\section{SUMMARY}

We have created median and geometric mean composite quasar spectra using a sample of over 2200 quasars in the SDSS. The resolution and $\mathrm{S} / \mathrm{N}$ exceed all previously published UV-optical quasar composites. Over 80 emissionline features have been detected and identified. We have been able to measure velocity shifts in a large number of both permitted and forbidden emission-line peaks, most of which have no such previous measurements. Power-law fits to the continua verify the results from most recent studies. The composites show that there is a lack of emission-free regions across most of the UV-optical wavelength range, which makes fitting quasar continua difficult unless a very wide wavelength range is available.

The SDSS is rapidly producing high-quality spectra of quasars that cover a wide range of properties. Composite spectra can therefore be made from numerous subsamples in order to search for dependencies of global spectral characteristics on a variety of quasar parameters, such as redshift, luminosity, and radio loudness - a program that is currently underway. We are also using other techniques, such as principal component analysis, to examine trends among the diversity of quasar spectra.

The median composite is being used as a crosscorrelation template for spectra in the SDSS, and many other applications are imaginable. The median composite spectrum is likely to be of general interest, so it is available as a machine-readable table (Table 1).

The SDSS $^{30}$ is a joint project of the University of Chicago, Fermilab, the Institute for Advanced Study, the Japan Participation Group, Johns Hopkins University, the Max-Planck-Institute für Astronomie, New Mexico State University, Princeton University, the US Naval Observatory, and the University of Washington. Apache Point Observatory, site of the SDSS telescopes, is operated by the Astrophysical Research Consortium. Funding for the project has been provided by the Alfred P. Sloan Foundation, the SDSS member institutions, the National Aeronautics and Space Administration, the National Science Foundation, the US Department of Energy, Monbusho, and the Max Planck Society. We thank Simon Morris for making an electronic version of the LBQS composite quasar spectrum available to us and Bev Wills for helpful comments. M. A. S. acknowledges support from NSF grant AST 00-71091. D. P. S. and G. T. R. acknowledge support from NSF grant AST 99-0703. X. F. acknowledges support from NSF grant PHY 00-70928 and a Frank and Peggy Taplin Fellowship.

\footnotetext{
${ }^{30}$ The SDSS Web site is http://www.sdss.org/.
} 
Appenzeller, I., \& Wagner, S. J. 1991, A\&A, 250, 57

Bajtlik, S., Duncan, R. C., \& Ostriker, J. P. 1988, ApJ, 327, 570

Becker, R. H., White, R. L., \& Helfand, D. J. 1995, ApJ, 450, 559

Blanton, M., et al. 2001, AJ, 121, 2358

Boroson, T. A., \& Green, R. F. 1992, ApJS, 80, 109

Brotherton, M. S., Tran, H. T., Becker, R. H., Gregg, M. D., LaurentMuehleisen, S. L., \& White, R. L. 2001, ApJ, 546, 775

Brotherton, M. S., Wills, B. J., Francis, P. J., \& Steidel, C. C. 1994a, ApJ, 430, 495

Brotherton, M. S., Wills, B. J., Steidel, C. C., \& Sargent, W. L. W. 1994b, ApJ, 423, 131

Carballo, R., González-Serrano, J. I., Benn, C. R., Sánchez, S. F., \& Vigotti, M. 1999, MNRAS, 306, 137

Cid Fernandes, R. J., \& Terlevich, R. 1995, MNRAS, 272, 423

Corbin, M. R. 1990, ApJ, 357, 346

. 1995, ApJ, 447, 496

1997, ApJ, 485, 517

Cristiani, S., \& Vio, R. 1990, A\&A, 227, 385

Efstathiou, A., Hough, J. H., \& Young, S. 1995, MNRAS, 277, 1134

Elvis, M., et al. 1994, ApJS, 95, 1

Espey, B., \& Andreadis, S. 1999, in ASP Conf. Ser. 162, Quasars and Cosmology, ed. G. Ferland \& J. Baldwin (San Francisco: ASP), 351

Espey, B. R., Carswell, R. F., Bailey, J. A., Smith, M. G., \& Ward, M. J. 1989, ApJ, 342, 666

Fan, X. et al. 2001, AJ, 121, 31 2000, AJ, 119,1
1999, AJ, 118,1

Foltz, C. B., Weymann, R. J., Peterson, B. M., Sun, L., Malkan, M. A., \& Chaffee, F. H. 1986, ApJ, 307, 504

Forster, K., Green, P. J., Aldcroft, T. L., Vestergaard, M., Foltz, C. B., \&

Hewett, P. C. 2001, ApJS, 134, 35

Francis, P. J. 1993, ApJ, 407, 519 1996, Publ. Astron. Soc. Australia, 13, 212

Francis, P. J., Hewett, P. C., Foltz, C. B., \& Chaffee, F. H. 1992, ApJ, 398, 476

Francis, P. J., Hewett, P. C., Foltz, C. B., Chaffee, F. H., Weymann, R. J., \& Morris, S. L. 1991, ApJ, 373, 465

Franx, M., Illingworth, G., \& Heckman, T. 1989, ApJ, 344, 613

Frieman, J. A., et al. 2001, in preparation

Fukugita, M., Ichikawa, T., Gunn, J. E., Doi, M., Shimasaku, K., \& Schneider, D. P. 1996, AJ, 111, 1748

Gaskell, C. M. 1982, ApJ, 263, 79

Graham, M. J., Clowes, R. G., \& Campusano, L. E. 1996, MNRAS, 279, 1349

Grandi, S. A. 1977, ApJ, 215, 446

1981, ApJ, 251, 451

1982, ApJ, 255, 25

Gunn, J. E., et al. 1998, AJ, 116, 3040

Heckman, T. M., Butcher, H. R., Miley, G. K., \& van Breugel, W. J. M. 1981, ApJ, 247, 403

Hutchings, J. B., Gower, A. C., \& Price, R. 1987, AJ, 93, 6

Kriss, G. A., Peterson, B. M., Crenshaw, D. M., \& Zheng, W. 2000, ApJ, 535,58

Laor, A., Bahcall, J. N., Jannuzi, B. T., Schneider, D. P., \& Green, R. F. 1995, ApJS, 99, 1

Laor, A., Jannuzi, B. T., Green, R. F., \& Boroson, T. A. 1997, ApJ, 489, 656

Lupton, R. H. 1993, Statistics in Theory and Practice (Princeton: Princeton Univ. Press)

Mathis, J. S., Rumpl, W., \& Nordsieck, K. H. 1977, ApJ, 217, 425
McIntosh, D. H., Rix, H.-W., Rieke, M. J., \& Foltz, C. B. 1999, ApJ, 517, L73

McLure, R. J., \& Dunlop, J. S. 2000, MNRAS, 317, 249

McLure, R. J., Kukula, M. J., Dunlop, J. S., Baum, S. A., O’Dea, C. P., \& Hughes, D. H. 1999, MNRAS, 308, 377

Menou, K., et al. 2001, ApJ, submitted (astro-ph/0102410)

Mirabel, I. F., \& Wilson, A. S. 1984, ApJ, 277, 92

Natali, F., Giallongo, E., Cristiani, S., \& La Franca, F. 1998, AJ, 115, 397

Netzer, H. 1977, MNRAS, 181, 89P

Netzer, H., \& Wills, B. J. 1983, ApJ, 275, 445

Neugebauer, G., Green, R. F., Matthews, K., Schmidt, M., Soifer, B. T., \& Bennett, J. 1987, ApJS, 63, 615

Newberg, H. J., Richards, G. T., Richmond, M., \& Fan, X. 1999, ApJS, 123, 377

Newberg, H. J., et al. 2001, in preparation

Nolan, L. A., Dunlop, J. S., Kukula, M. J., Hughes, D. H., Boroson, T., \& Jimenez, R. 2001, MNRAS, 323, 308

Oke, J. B., \& Lauer, T. R. 1979, ApJ, 230, 360

Oke, J. B., Shields, G. A., \& Korycansky, D. G. 1984, ApJ, 277, 64

Osterbrock, D. E. 1981, ApJ, 246, 696

Osterbrock, D. E., Tran, H. D., \& Veilleux, S. 1992, ApJ, 389, 305

Penston, M. V. 1977, MNRAS, 180, 27P

Penston, M. V., Fosbury, R. A. E., Boksenberg, A., Ward, M. J., \& Wilson, A. S. 1984, MNRAS, 208, 347

Phillips, M. M. 1976, ApJ, 208, 37

. 1978, ApJS, 38, 187

Pitman, K. M., Clayton, G. C., \& Gordon, K. D. 2000, PASP, 112, 537

Puget, J. L., Leger, A., \& Boulanger, F. 1985, A\&A, 142, L19

Richards, G. T., et al. 2001a, in preparation

2001b, AJ, 121, 2308

Schlegel, D. J., Finkbeiner, D. P., \& Davis, M. 1998, ApJ, 500, 525

Schneider, D. P., Schmidt, M., \& Gunn, J. E. 1991, AJ, 101, 2004

Schneider, D. P., et al. 2001, AJ, 121, 1232

. 2000, PASP, 112,6

Serote Roos, M., Boisson, C., Joly, M., \& Ward, M. J. 1998, MNRAS, 301, 1

Siegmund, W., et al. 2001, in preparation

Snijders, M. A. J., Netzer, H., \& Boksenberg, A. 1986, MNRAS, 222, 549

Stoughton, C., et al. 2001, in preparation

Tadhunter, C. N., Metz, S., \& Robinson, A. 1994, MNRAS, 268, 989

Terlevich, E., Diaz, A. I., \& Terlevich, R. 1990, MNRAS, 242, 271

Tytler, D., \& Fan, X. 1992, ApJS, 79, 1

Uomoto, A., et al. 2001, in preparation

Vanden Berk, D. E., et al. 2001, in preparation

Verner, E. M., Verner, D. A., Korista, K. T., Ferguson, J. W., Hamann, F. \& Ferland, G. J. 1999, ApJS, 120, 101

Vrtilek, J. M., \& Carleton, N. P. 1985, ApJ, 294, 106

Weymann, R. J., Morris, S. L., Foltz, C. B., \& Hewett, P. C. 1991, ApJ, 373, 23

Whittle, M. 1985, MNRAS, 216, 817

Wilkes, B. J. 1986, MNRAS, 218, 331

Wills, B. J., Laor, A., Brotherton, M. S., Wills, D., Wilkes, B. J., Ferland, G. J., \& Shang, Z. 1999, ApJ, 515, L53

Wills, B. J., Netzer, H., \& Wills, D. 1985, ApJ, 288, 94

York, D. G., et al. 2000, AJ, 120, 1579

Zheng, W., Kriss, G. A., Telfer, R. C., Grimes, J. P., \& Davidsen, A. F. 1997, ApJ, 475, 469

Zheng, W., \& Sulentic, J. W. 1990, ApJ, 350, 512

Zheng, W., et al. 2000, AJ, 120, 1607 